\title{
VARIATIONAL ANALYSIS FOR THE BLACK AND SCHOLES EQUATION WITH STOCHASTIC VOLATILITY
}

\author{
Yves Achdou $^{1}$ AND Nicoletta TCHOU ${ }^{2}$
}

\begin{abstract}
We propose a variational analysis for a Black and Scholes equation with stochastic volatility. This equation gives the price of a European option as a function of the time, of the price of the underlying asset and of the volatility when the volatility is a function of a mean reverting OrsteinUhlenbeck process, possibly correlated with the underlying asset. The variational analysis involves weighted Sobolev spaces. It enables to prove qualitative properties of the solution, namely a maximum principle and additional regularity properties. Finally, we make numerical simulations of the solution, by finite element and finite difference methods.
\end{abstract}

Mathematics Subject Classification. 91B28, 91B24, 35K65, 65M06, 65M60.

Received: May 9, 2001. Revised: February 4, 2002.

\section{INTRODUCTION}

We consider a financial asset whose price is given by the stochastic differential equation

$$
\mathrm{d} X_{t}=\mu X_{t} \mathrm{~d} t+\sigma_{t} X_{t} \mathrm{~d} W_{t}
$$

where $\mu X_{t} \mathrm{~d} t$ is a drift term, $\left(W_{t}\right)$ is a Brownian motion, and $\left(\sigma_{t}\right)$ is the volatility. The simplest models (see [16]) for a complete overview) take a constant volatility, but these models are generally to rough to match real prices. A more realistic model consists in assuming that $\left(\sigma_{t}\right)$ is a function of a mean reverting Orstein-Uhlenbeck process:

$$
\begin{aligned}
& \sigma_{t}=f\left(Y_{t}\right), \\
& \mathrm{d} Y_{t}=\alpha\left(m-Y_{t}\right) \mathrm{d} t+\beta \mathrm{d} \hat{Z}_{t},
\end{aligned}
$$

where $\alpha, m$ and $\beta$ are positive constants, and where $\left(\hat{Z}_{t}\right)$ is a Brownian motion. As explained in [5], the law of $Y_{t}$ knowing $Y_{0}$ is $\mathcal{N}\left(m+\left(Y_{0}-m\right) \mathrm{e}^{-\alpha t}, \frac{\beta^{2}}{\alpha}\left(1-\mathrm{e}^{-2 \alpha t}\right)\right)$. Therefore, $m$ is the limit of the mean value of $Y_{t}$ as $t \rightarrow+\infty$, and $\frac{1}{\alpha}$ is the characteristic time of mean reversion. The parameter $\alpha$ is called the rate of mean

Keywords and phrases. Degenerate parabolic equations, european options, weighted Sobolev spaces, finite element and finite difference method.

1 UFR Mathématiques, Université Paris 7, 2 Place Jussieu, 75252 Paris cedex 5, France. Laboratoire d'Analyse Numérique,

Université Paris 6. e-mail: achdou@math.jussieu.fr

2 IRMAR, Université de Rennes 1, Rennes, France. 
reversion. The ratio $\frac{\beta^{2}}{\alpha}$ is the limit of the variance of $Y_{t}$ as $t \rightarrow+\infty$. For convenience, we introduce the parameter $\nu$

$$
\nu^{2}=\frac{\beta^{2}}{2 \alpha}
$$

The Brownian motion $\hat{Z}_{t}$ may be correlated with $W_{t}$ : it can be written as a linear combination of $\left(W_{t}\right)$ and an independent Brownian motion $\left(Z_{t}\right)$ :

$$
\hat{Z}_{t}=\rho W_{t}+\sqrt{1-\rho^{2}} Z_{t}
$$

where the correlation factor $\rho$ lies in $[-1,1]$.

Consider a European derivative on this asset, with expiration date $T$ and payoff function $h\left(X_{T}\right)$. Its price at the time $t$ will depend on $t$, on the price of the underlying asset $X_{t}$, and on $Y_{t}$. We denote by $P\left(t, X_{t}, Y_{t}\right)$ the price of the derivative, and by $\tilde{r}(t)$ the interest rate. By using the no arbitrage principle and the two dimensional Itô's formula, it is possible to prove that there exists a function $\tilde{\gamma}$ such that the pricing function $P$ satisfies the partial differential equation

$$
\begin{aligned}
& \frac{\partial P}{\partial t}+\frac{1}{2} f(y)^{2} x^{2} \frac{\partial^{2} P}{\partial x^{2}}+\rho \beta x f(y) \frac{\partial^{2} P}{\partial x \partial y}+\frac{1}{2} \beta^{2} \frac{\partial^{2} P}{\partial y^{2}} \\
& +\tilde{r}(t)\left(x \frac{\partial P}{\partial x}-P\right)+(\alpha(m-y)-\beta \tilde{\Lambda}(t, x, y)) \frac{\partial P}{\partial y}=0, \quad 0 \leq t<T, x>0, y \in \mathbb{R}
\end{aligned}
$$

where

$$
\tilde{\Lambda}(t, x, y)=\rho \frac{\mu-\tilde{r}(t)}{f(y)}+\sqrt{1-\rho^{2}} \tilde{\gamma}(t, x, y),
$$

with the terminal condition $P(T, x, y)=h(x)$. The function $\tilde{\gamma}(t, x, y)$ can be chosen arbitrarily.

The no arbitrage argument can be summarized as follows: we look for the pricing function $P$ by trying to construct a hedged portfolio of assets. It is not sufficient to hedge only with the underlying asset because there are two independent sources of randomness: $\mathrm{d} W_{t}$ and $\mathrm{d} Z_{t}$. So the idea is to take a self-financing hedged portfolio containing $a_{t}$ shares of the underlying asset, one option with expiration date $T_{1}$ whose price is

$$
P_{t}^{(1)}=P^{(1)}\left(t, X_{t}, Y_{t}\right)
$$

and $b_{t}$ options with a larger expiration date $T_{2}>T_{1}$, whose price is

$$
P_{t}^{(2)}=P^{(2)}\left(t, X_{t}, Y_{t}\right)
$$

The value of the portfolio is $c_{t}$. The no arbitrage principle yields that for $t<T_{1}$,

$$
\mathrm{d} c_{t}=a_{t} \mathrm{~d} X_{t}+\mathrm{d} P_{t}^{(1)}+b_{t} \mathrm{~d} P_{t}^{(2)}=\tilde{r}_{t} c_{t} \mathrm{~d} t=\tilde{r}_{t}\left(a_{t} X_{t}+P_{t}^{(1)}+b_{t} P_{t}^{(2)}\right) \mathrm{d} t .
$$

The two-dimensional Itô formula permits to write $\mathrm{d} P_{t}^{(1)}$ and $\mathrm{d} P_{t}^{(2)}$ as combinations of $\mathrm{d} t, \mathrm{~d} W_{t}$ and $\mathrm{d} Z_{t}$. The left hand side of (7) does not contain $\mathrm{d} Z_{t}$ so

$$
b_{t}=-\frac{\frac{\partial P^{(2)}}{\partial y}}{\frac{\partial P^{(1)}}{\partial y}} .
$$


From the last equation and since the left hand side of (7) does not contain $\mathrm{d} W_{t}$, we have also

$$
a_{t}+\frac{\partial P^{(1)}}{\partial x}+b_{t} \frac{\partial P^{(2)}}{\partial x}=0
$$

Comparing the $\mathrm{d} t$ terms in (7) and substituting the values of $a_{t}$ and $b_{t}$, we obtain that

$$
\begin{aligned}
\left.\frac{1}{\frac{\partial P^{(1)}}{\partial y}\left(\frac{\partial P^{(1)}}{\partial t}+\right.}+\frac{1}{2} f(y)^{2} x^{2} \frac{\partial^{2} P^{(1)}}{\partial x^{2}}+\rho \beta x f(y) \frac{\partial^{2} P^{(1)}}{\partial x \partial y}+\frac{1}{2} \beta^{2} \frac{\partial^{2} P^{(1)}}{\partial y^{2}}+\tilde{r}(t)\left(x \frac{\partial P^{(1)}}{\partial x}-P^{(1)}\right)\right)= \\
\frac{1}{\frac{\partial P^{(2)}}{\partial y}}\left(\frac{\partial P^{(2)}}{\partial t}+\frac{1}{2} f(y)^{2} x^{2} \frac{\partial^{2} P^{(2)}}{\partial x^{2}}+\rho \beta x f(y) \frac{\partial^{2} P^{(2)}}{\partial x \partial y}+\frac{1}{2} \beta^{2} \frac{\partial^{2} P^{(2)}}{\partial y^{2}}+\tilde{r}(t)\left(x \frac{\partial P^{(2)}}{\partial x}-P^{(2)}\right)\right) .
\end{aligned}
$$

In the equation above, the left hand side does not depend on $T_{2}$ and the right hand side does not depend on $T_{1}$, so there exists a function of $g(t, x, y)$ such that

$$
\frac{1}{\frac{\partial P}{\partial y}}\left(\frac{\partial P}{\partial t}+\frac{1}{2} f(y)^{2} x^{2} \frac{\partial^{2} P}{\partial x^{2}}+\rho \beta x f(y) \frac{\partial^{2} P}{\partial x \partial y}+\frac{1}{2} \beta^{2} \frac{\partial^{2} P}{\partial y^{2}}+\tilde{r}(t)\left(x \frac{\partial P}{\partial x}-P\right)\right)=g(t, x, y) .
$$

Choosing to write $g(t, x, y)=\alpha(y-m)+\beta \tilde{\lambda}(t, x, y)$ permits to make the infinitesimal generator of the OrsteinUhlenbeck process appear explicitly in the last equation and to obtain (5), so that as explained in [5], we can group the differential operator in (5) as follows:

$$
\underbrace{\frac{\partial P}{\partial t}+\frac{1}{2} f(y)^{2} x^{2} \frac{\partial^{2} P}{\partial x^{2}}+\tilde{r}(t)\left(x \frac{\partial P}{\partial x}-P\right)}_{B S_{f(y)}}+\underbrace{\rho \beta x f(y) \frac{\partial^{2} P}{\partial x \partial y}}_{\text {correlation }}+\underbrace{\frac{1}{2} \beta^{2} \frac{\partial^{2} P}{\partial y^{2}}+\alpha(m-y) \frac{\partial P}{\partial y}}_{\text {Orstein Uhlenbeck }}-\underbrace{\beta \tilde{\Lambda}(t, x, y) \frac{\partial P}{\partial y}}_{\text {premium }} .
$$

The premium term is the market price of the volatility risk: the reason to decompose $\tilde{\Lambda}$ as in (6) is that, in the perfectly correlated case $(|\rho|=1)$, it is possible to find the equation satisfied by $P$ by a simpler no arbitrage argument with a hedged portfolio containing only the option and shares of the underlying assets. In this case, the equation found for $P$ is:

$$
\begin{aligned}
\frac{\partial P}{\partial t}+\frac{1}{2} f(y)^{2} x^{2} \frac{\partial^{2} P}{\partial x^{2}} & +\rho \beta x f(y) \frac{\partial^{2} P}{\partial x \partial y}+\frac{1}{2} \beta^{2} \frac{\partial^{2} P}{\partial y^{2}} \\
& +\tilde{r}(t)\left(x \frac{\partial P}{\partial x}-P\right)+\left(\alpha(m-y)-\beta \rho \frac{\mu-\tilde{r}(t)}{f(y)}\right) \frac{\partial P}{\partial y}=0, \quad 0 \leq t<T, x>0, y \in \mathbb{R} .
\end{aligned}
$$

The term $\frac{\mu-\tilde{r}(t)}{f(y)}$ is called the excess return to risk ratio.

Finally, with (5), the Itô formula and (6)

$$
\mathrm{d} P\left(t, X_{t}, Y_{t}\right)=\left(x f(y) \frac{\partial P}{\partial x}+\beta \rho \frac{\partial P}{\partial y}\right)\left(\frac{\mu-\tilde{r}}{f(y)} \mathrm{d} t+\mathrm{d} W_{t}\right)+\beta \sqrt{1-\rho^{2}} \frac{\partial P}{\partial y}\left(\tilde{\gamma} \mathrm{d} t+\mathrm{d} Z_{t}\right)
$$

so we see that the function $\tilde{\gamma}$ is the contribution of the second source of randomness $\mathrm{d} Z_{t}$ to the risk premium.

There remains to choose the function $f$. In [14], Stein and Stein have considered the case when

$$
f(y)=|y| .
$$

In the following, we will study the equation (5) for $f(y)=|y|$ then for more general functions. 
We will assume hereafter that $\tilde{\gamma}$ is a bounded function. This assumption is too restrictive: indeed, with (10), the results below can be generalized to the case when $|\tilde{\gamma}(t, x, y)|$ is only bounded by $\frac{C}{|y|}$ when $y \rightarrow 0$, so $\tilde{\Lambda}$ may blow up at $y=0$. However, even with $\tilde{\gamma}$ bounded, the premium term still contains the term $\rho \frac{\mu-\tilde{r}(t)}{|y|} P$, so the difficulty of a singular premium term as $y \rightarrow 0$ will be treated in this paper when $\rho \neq 0$.

With (10) and when $\rho \neq 0$, it is natural to impose that

$$
P(t, x, 0)=g(t, x), \quad 0 \leq t<T, x>0,
$$

where

$$
\frac{\partial g}{\partial t}+\tilde{r}(t)\left(x \frac{\partial g}{\partial x}-g\right)=0 \quad 0 \leq t<T, x>0, \quad g(t=T)=h,
$$

because the price of the option will not depend on the volatility near $y=0$ ( $y \sim 0$ and $\frac{\partial P}{\partial y} \sim 0$ because $\Lambda$ blows up); so in this case, it is possible to consider two independent boundary value problems in $\left[0, T\left[\times \mathbb{R}_{+} \times \mathbb{R}_{+}\right.\right.$and $\left[0, T\left[\times \mathbb{R}_{+} \times \mathbb{R}_{-}\right.\right.$, with the Dirichlet conditions (11).

To our knowledge, no variational analysis has been supplied yet for these boundary value problems. We believe that such an analysis gives the correct setting to study the qualitative properties of the solutions, to justify rigorously asymptotic expansions when $\alpha$ is large (see [5]), and to find error estimates for numerical schemes.

In a first part we describe the changes of variables and unknown that will lead us to the variational formulation. In the second part, we introduce some suitable weighted Sobolev spaces and prove the continuity of the operator in these spaces. In the third part, we focus on the case when $\rho=0$, we prove existence for the weak formulation and qualitative results by means of a maximum principle adapted to our degenerate operator. In the fourth part we deal with $\rho \neq 0$. Then we generalize our approach to arbitrary functions $f$. In the last part, we give numerical results obtained by two different codes, with finite elements and a finite difference method.

In this paper, we will not discuss the problem of hedging, which consists in constructing a portfolio containing shares of the risk-less asset and of the underlying asset in order to hedge (i.e. replicate) the price of the option. This problem is of course very important for risk management. As shown in [5] and references therein, with the stochastic volatility model, it is not possible to hedge exactly the option with a self-financed portfolio; indeed, in order to hedge exactly the option, one has to inject money in the portfolio, so hedging has a cost, and one problem is to find a good trade-off between the risk due to approximate hedging and the cost of implementing the hedge. In any case, hedging implies an accurate knowledge of some partial derivatives of the pricing function and for that reason, it is a challenging problem for numerical computations.

\section{Change of variables And UnKnown}

To obtain a forward parabolic equation, we change the sense of time. Also, in order to use a variational method, we make the change of unknown

$$
u(t, x, y)=P(T-t, x, y) \mathrm{e}^{-(1-\eta) \frac{(y-m)^{2}}{2 \nu^{2}}},
$$

where $\eta$ is a parameter such that $0<\eta<1$, because it can be seen very easily that if $\tilde{\Lambda}=0$ and $\rho=0$, then the function $\mathrm{e}^{\frac{(y-m)^{2}}{2 \nu^{2}}}$ satisfies (5). If $P$ is a bounded solution of (5), the function $u$ should decay fast when $y$ goes to infinity: we will look for $u$ in a weighted Sobolev space (weak solution), and we shall prove afterward that the function $u$ obtained by this method has the correct behaviour at infinity, so that the function $u(T-t, x, y) \mathrm{e}^{(1-\eta) \frac{(y-m)^{2}}{2 \nu^{2}}}$ is a bounded solution of (5). 
With the notations $r(t)=\tilde{r}(T-t), \gamma(t)=\tilde{\gamma}(T-t)$ and $\Lambda(t)=\tilde{\Lambda}(T-t)$ the new unknown $u$ satisfies the degenerate parabolic partial differential equation

$$
\begin{aligned}
& \frac{\partial u}{\partial t}-\frac{1}{2} y^{2} x^{2} \frac{\partial^{2} u}{\partial x^{2}}-r(t)\left(x \frac{\partial u}{\partial x}-u\right)-\rho \beta x|y| \frac{\partial^{2} u}{\partial x \partial y}+2 \rho \frac{\alpha}{\beta}(m-y) x|y| \frac{\partial u}{\partial x} \\
& -\frac{1}{2} \beta^{2} \frac{\partial^{2} u}{\partial y^{2}}+(-\alpha(y-m)+\beta \Lambda(t, x, y)) \frac{\partial u}{\partial y}+\left(2 \frac{\alpha}{\beta} \Lambda(t, x, y)(y-m)-\alpha\right) u \\
& +\eta\left(2 \alpha(y-m) \frac{\partial u}{\partial y}+2 \rho \frac{\alpha}{\beta}(y-m) x|y| \frac{\partial u}{\partial x}+2 \frac{\alpha^{2}}{\beta^{2}}(1-\eta)(y-m)^{2} u-2 \frac{\alpha}{\beta} \Lambda(y-m) u+\alpha u\right)=0
\end{aligned}
$$

or, by expanding $\Lambda$ and by denoting by $\mathcal{L}_{t, \rho}$ the linear partial differential operator

$$
\begin{aligned}
& \mathcal{L}_{t, \rho}(v)=-\frac{1}{2} y^{2} x^{2} \frac{\partial^{2} v}{\partial x^{2}}-\frac{1}{2} \beta^{2} \frac{\partial^{2} v}{\partial y^{2}}-\rho \beta x|y| \frac{\partial^{2} v}{\partial x \partial y}+\left(-r(t)-2 \rho(1-\eta) \frac{\alpha}{\beta}(y-m)|y|\right) x \frac{\partial v}{\partial x} \\
& +\left(-(1-2 \eta) \alpha(y-m)+\beta\left(\rho \frac{\mu-r(t)}{|y|}+\sqrt{1-\rho^{2}} \gamma(t, x, y)\right)\right) \frac{\partial v}{\partial y} \\
& +\left(r(t)+2 \frac{\alpha^{2}}{\beta^{2}} \eta(1-\eta)(y-m)^{2}+2(1-\eta) \frac{\alpha}{\beta}(y-m)\left(\rho \frac{\mu-r(t)}{|y|}+\sqrt{1-\rho^{2}} \gamma(t, x, y)\right)-\alpha(1-\eta)\right) v,
\end{aligned}
$$

we obtain

$$
\frac{\partial u}{\partial t}+\mathcal{L}_{t, \rho} u=0
$$

\section{VARIATIONAL FORMULATION}

\subsection{Some weighted Sobolev spaces}

We denote by $Q$ the open half plane $Q=\mathbb{R}_{+} \times \mathbb{R}$, and by $Q_{ \pm}$the open quarter of plane $Q_{ \pm}=\mathbb{R}_{+} \times \mathbb{R}_{ \pm}$. Let us consider the weighted Sobolev space $V$ :

$$
V=\left\{v:\left(\sqrt{1+y^{2}} v, \frac{\partial v}{\partial y}, x|y| \frac{\partial v}{\partial x}\right) \in\left(L^{2}(Q)\right)^{3}\right\} .
$$

This space with the norm

$$
\||v|\|_{V}=\left(\int_{Q}\left(1+y^{2}\right) v^{2}+\left(\frac{\partial v}{\partial y}\right)^{2}+x^{2} y^{2}\left(\frac{\partial v}{\partial x}\right)^{2}\right)^{\frac{1}{2}}
$$

is a Hilbert space, and it has the following properties:

1. $V$ is separable and reflexive. We call $V^{\prime}$ the dual of $V$,

2. $\mathcal{D}(Q) \subset V$ and $\mathcal{D}(Q)$ is dense in $V$,

3. $V$ is dense in $L^{2}(Q)$.

In order to prove the point 2 , we first make the change of variable $(z, y)=(\log (x), y)$, so $v \in V \Leftrightarrow \tilde{v} \in \tilde{V}$ where $\tilde{v}(z, y)=v(\exp (z), y)$ and

$$
\tilde{V}=\left\{w:\left(\sqrt{1+y^{2}} w \exp (z / 2), \frac{\partial w}{\partial y} \exp (z / 2),|y| \frac{\partial w}{\partial z} \exp (z / 2)\right) \in\left(L^{2}\left(\mathbb{R}^{2}\right)\right)^{3}\right\}
$$


Proving the point 2 is equivalent to proving that $\mathcal{C}_{0}^{\infty}\left(\mathbb{R}^{2}\right)$ is dense in $\tilde{V}$, equipped with the norm $\|w\|_{\tilde{V}}=$ $\left(\int_{\mathbb{R}^{2}} \exp (z)\left(\left(1+y^{2}\right) w^{2}+\left(\frac{\partial w}{\partial y}\right)^{2}+y^{2}\left(\frac{\partial w}{\partial z}\right)^{2}\right)\right)^{\frac{1}{2}}$. But this statement is a consequence of a eneral result by Friedrichs (Th. 4.2 in $[8])$.

We also have the estimate

Lemma 1. Let $v$ be a function in $V$. Then

$$
\int_{Q} y^{2} v^{2} \leq 4 \int_{Q} y^{2} x^{2}\left(\frac{\partial v}{\partial x}\right)^{2}
$$

so the semi-norm

$$
\|v\|_{V}=\left(\int_{Q} v^{2}+\left(\frac{\partial v}{\partial y}\right)^{2}+x^{2} y^{2}\left(\frac{\partial v}{\partial x}\right)^{2}\right)^{\frac{1}{2}}
$$

is in fact a norm in $V$, equivalent to $\||\cdot|\|$.

Proof. It is enough to prove (19) for functions in $\mathcal{D}(Q)$. Let $v$ be a function in $\mathcal{D}(Q)$, we have that

$$
\int_{Q} y^{2} v^{2}=-2 \int_{Q} x y^{2} \frac{\partial v}{\partial x} v
$$

and the result stems from the Cauchy-Schwarz inequality.

Remark 1. The inequality (19) holds when $Q$ is replaced with $\mathbb{R}_{+} \times I$ and $I$ is any interval.

It is also possible to define $V_{0, \pm}$ as the closure of $\mathcal{D}\left(Q_{ \pm}\right)$in $V$ and we call $V_{0, \pm}^{\prime}$ the dual of $V_{0, \pm}$. We have the Hardy inequality:

$$
\forall v \in V_{0, \pm}, \quad\left\|\frac{v}{y}\right\|_{L^{2}\left(Q_{ \pm}\right)} \leq 2\left\|\frac{\partial v}{\partial y}\right\|_{L^{2}\left(Q_{ \pm}\right)} .
$$

Lemma 2. The operator $v \rightarrow \beta x \frac{\partial v}{\partial x}$ is continuous from $V$ into $V^{\prime}$, and from $V_{0, \pm}$ into $V_{0, \pm}^{\prime}$.

Proof. Call $X$ and $Y$ the differential operators

$$
X(v)=x y \frac{\partial v}{\partial x}+\beta \frac{\partial v}{\partial y}, \quad Y(v)=x y \frac{\partial v}{\partial x}-\beta \frac{\partial v}{\partial y},
$$

The operators $X$ and $Y$ are continuous operators from $V$ into $L^{2}(Q)$ and their adjoints are

$$
X^{T}(v)=-x y \frac{\partial v}{\partial x}-\beta \frac{\partial v}{\partial y}-y v=-X(v)-y v, \quad Y^{T} v=-x y \frac{\partial v}{\partial x}+\beta \frac{\partial v}{\partial y}-y v=-Y(v)-y v .
$$

Consider the commutator $[X, Y]=X Y-Y X$ : it can be checked that

$$
[X, Y](v)=2 \beta x \frac{\partial v}{\partial x} .
$$

Therefore, for $v \in V$ and $w \in \mathcal{D}(Q)$,

$$
\left\langle 2 \beta x \frac{\partial v}{\partial x}, w\right\rangle=-\int_{Q} Y(v)(X(w)+y w)+\int_{Q} X(v)(Y(w)+y w),
$$


and from (19), there exists a constant $C$ such that

$$
\left|\left\langle 2 \beta x \frac{\partial v}{\partial x}, w\right\rangle\right| \leq C\|v\|_{V}\|w\|_{V} .
$$

To conclude, we use the density of $\mathcal{D}(Q)$ into $V$. The proof of the second assertion is identical.

Proposition 1. Assume that $r$ is a bounded function of time and that $\gamma$ is bounded by a constant. The operator $\mathcal{L}_{t, 0}$ is a bounded linear operator from $V$ into $V^{\prime}$ with a constant independent of $t$.

Proof. Consider $\left\langle\mathcal{L}_{t, 0} v, w\right\rangle$ for $v \in V$ and $w \in \mathcal{D}(Q)$. We have to prove that for a constant $C,\left|\left\langle\mathcal{L}_{t, 0} v, w\right\rangle\right| \leq$ $C\|v\|_{V}\|w\|_{V}$. We consider all the terms in (15) separately. The only term for which the continuity does not stem from an integration by part, a Cauchy-Schwarz inequalitiy and possibly Lemma 1, is the term $\left\langle-r(t) x \frac{\partial v}{\partial x}, w\right\rangle$; but from Lemma 2, the operator $x \frac{\partial v}{\partial x}$ is continuous from $V$ into $V^{\prime}$.

For the second assertion of the Proposition 1, we proceed in the same way, but we need also the Hardy inequality (21).

\subsection{The uncorrelated case}

Proposition 2. Assume that $r$ is a bounded function of time and that $\gamma$ is bounded by a constant $\Gamma$. Assume that $\alpha>\beta$, then there exist two positive constants $C$ and $c$ independent on $t$ and two constants $0<\eta_{1}<\eta_{2}<1$ such that, for $\eta_{1}<\eta<\eta_{2}$ and for any $v \in V$,

$$
\left\langle\mathcal{L}_{t, 0} v, v\right\rangle \geq C\|v\|_{V}^{2}-c\|v\|_{L^{2}(Q)}^{2} .
$$

Proof. We can write

$$
\left\langle\mathcal{L}_{t, 0} v, v\right\rangle=\sum_{i=1}^{6} \mathcal{I}_{i}
$$

with

$$
\begin{gathered}
\mathcal{I}_{1}=-\frac{1}{2}\left\langle x^{2} y^{2} \frac{\partial^{2} v}{\partial x^{2}} v\right\rangle, \quad \mathcal{I}_{2}=-\frac{\beta^{2}}{2}\left\langle\frac{\partial^{2} v}{\partial y^{2}}, v\right\rangle, \quad \mathcal{I}_{3}=-r(t)\left\langle x \frac{\partial v}{\partial x}, v\right\rangle, \\
\mathcal{I}_{4}=\int_{Q}(-\alpha(1-2 \eta)(y-m)+\beta \gamma(t, x, y)) v \frac{\partial v}{\partial y}, \\
\mathcal{I}_{5}=\int_{Q}\left(r(t)+2(1-\eta) \frac{\alpha}{\beta}(y-m) \gamma(t, x, y)-\alpha(1-\eta)\right) v^{2}, \\
\mathcal{I}_{6}=2 \frac{\alpha^{2}}{\beta^{2}} \eta(1-\eta) \int_{Q}(y-m)^{2} v^{2}, \\
\mathcal{I}_{1}=\frac{1}{2} \int_{Q} x^{2} y^{2}\left(\frac{\partial v}{\partial x}\right)^{2}+\int_{Q} x y^{2} v \frac{\partial v}{\partial x}=\frac{1}{2} \int_{Q} x^{2} y^{2}\left(\frac{\partial v}{\partial x}\right)^{2}+\frac{1}{2} \int_{Q} x y^{2} \frac{\partial v^{2}}{\partial x}=\frac{1}{2} \int_{Q} x^{2} y^{2}\left(\frac{\partial v}{\partial x}\right)^{2}-\frac{1}{2} \int_{Q} y^{2} v^{2}, \\
\mathcal{I}_{2}=\frac{\beta^{2}}{2} \int_{Q}\left(\frac{\partial v}{\partial y}\right)^{2}, \quad \mathcal{I}_{3}=\frac{r(t)}{2} \int_{Q} v^{2}, \\
\mathcal{I}_{4}=\frac{\alpha}{2}(1-2 \eta) \int_{Q} v^{2}+\beta \int_{Q} \gamma(t, x, y) v \frac{\partial v}{\partial y} \geq \frac{\alpha}{2}(1-2 \eta) \int_{Q} v^{2}-\frac{1}{2} \beta \Gamma\left(\zeta\|v\|_{L^{2}(Q)}^{2}+\frac{1}{\zeta}\left\|\frac{\partial v}{\partial y}\right\|_{L^{2}(Q)}^{2}\right),
\end{gathered}
$$


where $\zeta$ is an arbitrary positive number. Similarly,

$$
\begin{aligned}
\mathcal{I}_{5} & =(r(t)-\alpha(1-\eta))\|v\|_{L^{2}(Q)}^{2}+2(1-\eta) \frac{\alpha}{\beta} \int_{Q}(y-m) \gamma(t, x, y) v^{2} \\
& \geq\left(r(t)-\alpha(1-\eta)-2(1-\eta) \frac{\alpha}{\beta} \Gamma\left(|m|+\frac{1}{2} \zeta\right)\right)\|v\|_{L^{2}(Q)}^{2}-(1-\eta) \frac{\alpha \Gamma}{\zeta \beta}\|y v\|_{L^{2}(Q)}^{2}
\end{aligned}
$$

and

$$
\mathcal{I}_{6} \geq 2 \frac{\alpha^{2}}{\beta^{2}} \eta(1-\eta)\left(\|y v\|_{L^{2}(Q)}^{2}+m^{2}\|v\|_{L^{2}(Q)}^{2}-|m|\left(\zeta\|v\|_{L^{2}(Q)}^{2}+\frac{1}{\zeta}\|y v\|_{L^{2}(Q)}^{2}\right)\right) .
$$

If $\alpha>\beta$, it is possible to choose $C, 0<C<\frac{1}{2}$ and $\eta_{1}, \eta_{2}, 0<\eta_{1}<\eta_{2}<1$, such that for any $\eta$ with $\eta_{1}<\eta<\eta_{2}$ and for $\zeta$ large enough

$$
2 \frac{\alpha^{2}}{\beta^{2}} \eta(1-\eta)\left(1-\frac{|m|}{\zeta}\right)-\frac{1}{2}-(1-\eta) \frac{\alpha \Gamma}{\zeta \beta}>C
$$

and at the same time

$$
\frac{\beta}{2}\left(\beta-\frac{\Gamma}{\zeta}\right)>C
$$

Then the estimate (26) follows by fixing $\zeta$.

Consider now the pricing problem for a European vanilla put, whose payoff function is $P_{0}(x, y)=(x-K)_{-}$. After the change of unknown (13), the initial condition for $u$ is

$$
u(0, x, y)=u_{0}(x, y)=(x-K){ }_{-} \mathrm{e}^{-(1-\eta) \frac{(y-m)^{2}}{2 \nu^{2}}} .
$$

More generally, it is possible to consider any payoff function such that the corresponding $u_{0}$ belongs to $L^{2}(Q)$. The boundary value problem for $u$ is (16), with (27).

Theorem 1. Assume that $\alpha>\beta$ and that $\eta$ has been chosen as in Proposition 2. Then there exists a unique $u$ in $L^{2}(0, T ; V) \cap \mathcal{C}^{0}\left([0, T] ; L^{2}(Q)\right)$, with $\frac{\partial u}{\partial t} \in L^{2}\left(0, T ; V^{\prime}\right)$ such that, in the sense of the distributions in time,

$$
\frac{\mathrm{d}}{\mathrm{d} t}(u, v)+\left\langle\mathcal{L}_{t, 0} u, v\right\rangle=0 \quad \forall v \in V
$$

and

$$
u(t=0)=u_{0} .
$$

The mapping $u_{0} \mapsto u$ is continuous from $L^{2}(Q)$ to $L^{2}(0, T ; V) \cap \mathcal{C}^{0}\left([0, T] ; L^{2}(Q)\right)$. Furthermore, u satisfies (16) in the sense of distributions.

Proof. With Propositions 1 and 2, the proof comes from the general result of J.L. Lions and E. Magenes [2,9]. The fact that $u$ satisfies (16) in the sense of distributions comes from the density of $\mathcal{D}(Q)$ in $V$.

Remark 2. When Theorem 1 can be applied, the partial differential equation (16) holds in $L^{2}\left(0, T ; V^{\prime}\right)$.

Remark 3. The ratio $\frac{\alpha^{2}}{\beta^{2}}$ is exactly $\frac{\text { rate of mean reversion }}{\text { asymptotic variance of the volatility }}$. The assumption in Theorem 1 says that the rate of mean reversion should not too small compared with the asymptotic variance of the volatility. Moreover, the condition is usually satisfied in practice, since $\alpha$ is often much larger than the asymptotic variance $\frac{\beta^{2}}{\alpha}$. 
Remark 4. By using (19), it is possible to improve on the condition $\frac{\alpha}{\beta}>1$. Indeed, the condition $\frac{\alpha}{\beta}>\frac{\sqrt{3}}{2}$ is sufficient for proving Theorem 1.

Let us now discuss the special case when $r$ is constant. In this case, it is possible to use results on semigroups in order to get more information on $u$. So let us call $\left(D, A_{t}=-\mathcal{L}_{t, 0}\right)$ the family of unbounded operators in $L^{2}(Q)$ :

$$
\begin{aligned}
A_{t}= & A_{1}+A_{2, t} \\
A_{1} v= & \frac{1}{2} y^{2} x^{2} \frac{\partial^{2} v}{\partial x^{2}}+\frac{1}{2} \beta^{2} \frac{\partial^{2} v}{\partial y^{2}}+r x \frac{\partial v}{\partial x}-2 \frac{\alpha^{2}}{\beta^{2}} \eta(1-\eta) y^{2} v+\alpha(1-2 \eta) y \frac{\partial v}{\partial y} \\
A_{2, t} v= & -((1-2 \eta) m+\beta(\gamma(t, x, y))) \frac{\partial v}{\partial y} \\
& -\left(r+2 \frac{\alpha^{2}}{\beta^{2}} \eta(1-\eta)\left(-2 m y+m^{2}\right)+2(1-\eta) \frac{\alpha}{\beta}(y-m) \gamma(t, x, y)-\alpha(1-\eta)\right) v
\end{aligned}
$$

and $D$ is the domain of $A_{t}$ :

$$
D=\left\{v \in V ; A_{1} v \in L^{2}(Q)\right\}
$$

Note that $A_{2, t}$ is a bounded operator from $V$ to $L^{2}(Q)$, with a constant independent of $t$. It is clear that $D$ is dense in $L^{2}(Q)$ because $D$ contains $\mathcal{D}(Q)$.

From (26), there exists a constant $c>0$ large enough and a constant $C>0$ such that for all $t,((c I-$ $\left.\left.A_{t}\right) v, v\right)_{L^{2}(Q)} \geq C\|v\|_{V}^{2}$ for all $v$ in $D$. Thus the operator $\left(D,-c I+A_{t}\right)$ is dissipative in $L^{2}(Q)$, see $[2,3]$.

Also for all $f \in L^{2}(Q)$ the Lax-Milgram Theorem tells us that there exists a unique $u \in V$ such that $\left(c I+\mathcal{L}_{t, 0}\right) u=f$ and that $f \mapsto u$ is a bounded operator from $L^{2}(Q)$ to $V$, with a constant independent of $t$. This implies that $A_{1} u \in L^{2}(Q)$, therefore $u \in D$ and the mapping $f \mapsto u$ is bounded from $L^{2}(Q)$ to $D$, with a constant independent on $t$. Thus the graph of $-c I+A_{t}$ is closed, and the operator $-c I+A_{t}$ is m-dissipative, so if $t$ is fixed $-c I+A_{t}$ is the infinitesimal generator of a contraction semi-group, see [2,3]. Furthermore, since $A_{t}$ is a lower order perturbation of the constant in time operator $A_{1}$, it is possible to use the general results of Kato (see for example [10], Th. 3.1, p. 135).

Theorem 2. With the assumptions of Theorem 1, suppose that $r$ is constant and $\gamma$ belongs to $\mathcal{C}^{0}\left([0, T], L^{\infty}(Q)\right)$. If $u_{0}$ belongs to $D$ then the solution of (28) and (29) given by Theorem 1 belongs also to $\mathcal{C}^{1}\left((0, T) ; L^{2}(Q)\right) \cap$ $\mathcal{C}^{0}([0, T] ; D)$ and satisfies the equation

$$
\frac{\mathrm{d}}{\mathrm{d} t} u-A_{t} u=0
$$

for each $t \in(0, T]$.

From now on, we shall suppose that the assumptions of Theorems 1 and 2 are satisfied.

Remark 5. It is possible to prove the same result when the rate $r$ is a Lipschitz continuous function of time. For that, we need to study more precisely the domain of $A_{t}$. This will be the topic of a forthcoming work, [1]. In particular, if $r$ is bounded from below by a positive constant, and on slightly stronger condition on $\frac{\alpha}{\beta}$, it is possible to characterize the domain of $A_{t}$.

Remark 6. Unfortunately, the higher order part of the operator $A_{t}$ namely $A_{1}$ is not a lower order perturbation of an autodjoint operator. Therefore, it does not seem possible to use the results on analytic semi-groups (see $[3,10])$, and to prove a smoothing effect. 
With the assumptions of Theorem 1 , let us call $P$ the function

$$
P=u(T-t, x, y) \mathrm{e}^{(1-\eta) \frac{(y-m)^{2}}{2 \nu^{2}}}
$$

where $u$ is the solution of (28), (29). It is clear that $P$ is a solution of (5), (11) in a weak sense.

In order to prove bounds on $P$, we need to state a weak maximum principle: for this, we need to truncate the domain in the $y$-direction. We call $Q_{M}$ the half-strip $Q_{M}=\mathbb{R}_{+} \times(-M, M)$ and $V_{M}$ the Hilbert space

$$
V_{M}=\left\{v:\left(v, \frac{\partial v}{\partial y}, x|y| \frac{\partial v}{\partial x}\right) \in\left(L^{2}\left(Q_{M}\right)\right)^{3}\right\}
$$

and $V_{0, M}$ the completion of $\mathcal{D}\left(Q_{M}\right)$ in $V_{M}$.

Remark 7. The bound (26) holds in $V_{0, M}$, with the same constants.

Thanks to Remark 7, we obtain a similar result as Theorem 1, replacing $V$ by $V_{0, M}$ :

Lemma 3. There exists a unique function $u_{M}$ in $L^{2}\left(0, T ; V_{0, M}\right) \cap \mathcal{C}^{0}\left([0, T] ; L^{2}\left(Q_{M}\right)\right)$, with $\frac{\partial u_{M}}{\partial t} \in L^{2}\left(0, T ; V_{0, M}^{\prime}\right)$ such that, in the sense of the distributions

$$
\frac{\partial u_{M}}{\partial t}+\mathcal{L}_{t, 0} u_{M}=0
$$

and

$$
u_{M}(t=0, x, y)=u_{0}(x, y) .
$$

We extend $u_{M}$ by zero in $Q \backslash Q_{M}$ and we still call $u_{M}$ the extension. Thanks to Remark 7, its is possible to prove:

Lemma 4. Under the same assumptions as in Theorem 1, the sequence $u_{M}$ converges to the solution $u$ of (28), (29) in $L^{2}(0, T ; V) \cap \mathcal{C}^{0}\left([0, T] ; L^{2}(Q)\right)$, as $M \rightarrow+\infty$.

Lemma 5 (maximum principle). With the same assumptions as in Theorem 2, let $p$ be a function such that for any parameter $\epsilon>0, p \mathrm{e}^{-\epsilon \log ^{2}(x)} \in \mathcal{C}^{0}\left([0, T], L^{2}\left(Q_{M}\right)\right) \cap L^{2}\left(0, T ; V_{M}\right) \cap \mathcal{C}^{1}\left((0, T], L^{2}\left(Q_{M}\right)\right), \frac{\partial p}{\partial t} \mathrm{e}^{-\epsilon \log ^{2}(x)} \in$ $L^{2}\left(0, T ; V_{M}^{\prime}\right)$ and such that

$$
\begin{gathered}
p(0, x, y) \leq 0 \quad \text { a.e. } \\
p(t, x, \pm M) \leq 0
\end{gathered}
$$

and

$$
\frac{\partial p}{\partial t}+\mathcal{L}_{t, 0} p \leq 0
$$

in $\mathcal{D}^{\prime}\left((0, T) \times Q_{M}\right)$. Then $p(t, x, y) \leq 0$ a.e.

Proof. The proof is done by adapting an argument in [13]. It consists of taking $\mathrm{e}^{-\frac{\zeta}{2 T-t} \log ^{2}(x)-z t} p_{+} 1_{t>\tau}$ as a test-function in the equation satisfied by $p$, where $0<\zeta<1$, and $z>0$. For brevity, we call $\phi$ the function

$$
\phi(x, t)=\frac{\zeta}{2 T-t} \log ^{2}(x)-z t,
$$


and $\chi$ the function $\chi=\mathrm{e}^{-\frac{\phi}{2}} p_{+}$. After some computations, we obtain that

$$
\int_{\tau}^{t} \int_{Q_{M}} \frac{1}{2} \frac{\partial \chi^{2}}{\partial t}+\int_{\tau}^{t}\left\langle\mathcal{L}_{t, 0} \chi, \chi\right\rangle+\int_{\tau}^{t} \int_{Q_{M}} \kappa(t, x, y) \chi^{2} \leq 0
$$

where $\kappa$ is given by

$$
\kappa(t, x, y)=\frac{1}{2} z+\frac{\zeta^{2}}{2(2 T-t)^{2}} \log ^{2}(x)\left(\frac{1}{\zeta}-y^{2}\right)-\left(y^{2}-r\right) \frac{\zeta}{(2 T-t)} \log (x)+\xi(t, x, y)
$$

and $\xi$ is a bounded function, independent of $\zeta$ and $z$. We choose two values $x_{1}<1$ and $x_{2}>1$ such that, for $x \in\left(0, x_{1}\right) \cup\left(x_{2},+\infty\right), 4 T\left|y^{2}-r\right|<\frac{1}{2}|\log (x)|$. We call $\mathcal{S}_{1}$ the set $\mathcal{S}_{1}=\left(0, x_{1}\right) \cup\left(x_{2},+\infty\right)$ and $\mathcal{S}_{2}=\left[x_{1}, x_{2}\right]$

$$
\kappa(t, x, y) \geq \frac{\zeta}{2(2 T-t)^{2}} \log ^{2}(x)\left(1-\zeta y^{2}-4 T \max _{x \in \mathcal{S}_{1}} \frac{\left|y^{2}-r\right|}{|\log (x)|}\right)+\frac{1}{2} z-\max |\xi(t, x, y)|-\zeta \max _{x \in \mathcal{S}_{2}}\left|\frac{y^{2}-r}{(2 T-t)} \log (x)\right|
$$

But $4 T \max _{x \in \mathcal{S}_{1}} \frac{\left|y^{2}-r\right|}{|\log (x)|} \leq \frac{1}{2}$, so if we choose $\zeta$ small enough, $1-\zeta y^{2}-4 T \max _{x \in \mathcal{S}_{1}} \frac{\left|y^{2}-r\right|}{|\log (x)|} \geq 0$ for all $y \in$ $(-M, M)$. For such a $\zeta$, we choose $z$ large enough such that

$$
\left\langle\mathcal{L}_{t, 0} \chi, \chi\right\rangle+\int_{Q_{M}}\left(\frac{1}{2} z-\max |\xi(t, x, y)|-\zeta \max _{x \in \mathcal{S}_{2}}\left|\frac{y^{2}-r}{(2 T-t)} \log (x)\right|\right) \chi^{2} \geq 0
$$

for all values of $t$ in $(0, T)$. For these choices of $\zeta$ and $z$, the equation (35) implies that

$$
\frac{1}{2}\left(\|\chi(t)\|_{L^{2}\left(Q_{M}\right)}^{2}-\|\chi(\tau)\|_{L^{2}\left(Q_{M}\right)}^{2}\right) \leq 0
$$

Therefore, by letting $\tau$ tend to zero, we obtain that $\chi(t)=0$ a.e. in $x$, because $\chi(t=0)=0$ and $\chi \in$ $\mathcal{C}^{0}\left([0, T], L^{2}\left(Q_{M}\right)\right)$.

Proposition 3. Under the same assumptions as in Theorem 2, the function

$$
P=u(T-t, x, y) \mathrm{e}^{(1-\eta) \frac{(y-m)^{2}}{2 \nu^{2}}}
$$

where $u$ is the solution of (28), (29), satisfies

$$
\left(x-K \mathrm{e}^{-r(T-t)}\right)_{-} \leq P(t, x, y) \leq K \mathrm{e}^{-r(T-t)} .
$$

Proof. The proof is done with the maximum principle (Lem. 5). Let us prove the upper bound first: let us call $\chi_{M}$ the function $\chi_{M}=u_{M}-K \mathrm{e}^{-r t} \mathrm{e}^{-(1-\eta) \frac{(y-m)^{2}}{2 \nu^{2}}}$. It can be seen that $\chi_{M}(t=0) \leq 0$ and that $\frac{\partial \chi_{M}}{\partial t}+\mathcal{L}_{t, 0} \chi_{M}=0$ in the sense of distributions. Also, $\chi_{M}(t, x, \pm M) \leq 0$. To apply Lemma 5 , we need that $\frac{\partial \chi_{M}}{\partial t} \in \mathcal{C}^{0}\left((0, T], L^{2}\left(Q_{M}\right)\right)$, which is not true in general. However, it is possible to approximate $u_{0}$ in $L^{2}\left(Q_{M}\right)$ by a smooth function $\bar{u}_{0}$ vanishing near $y= \pm M$ and in the domain of the operator and such that $\bar{u}_{0} \leq \mathrm{e}^{-(1-\eta) \frac{(y-m)^{2}}{2 \nu^{2}}}$. Calling $\bar{u}_{M}$ the solution of the Cauchy problem with data $\bar{u}_{0}$, it is possible to apply Lemma 5 thanks to a result similar to Theorem 2, and to prove that a.e. $\bar{u}_{M}-K \mathrm{e}^{-r t} \mathrm{e}^{-(1-\eta) \frac{(y-m)^{2}}{2 \nu^{2}}} \leq 0$. Since the mapping $u_{0} \mapsto u$ is continuous from $L^{2}\left(Q_{M}\right)$ to $\mathcal{C}^{0}\left([0, T], L^{2}\left(Q_{M}\right)\right) \cap L^{2}\left([0, T], V_{0, M}\right)$, we also have $\chi_{M} \leq 0$ a.e.

The upper bound in (36) follows by passing to the limit when $M$ goes to infinity, thanks to Lemma 4. 
The lower bound is obtained in the same way: we call $\tilde{u}_{M}$ the function in $L^{2}\left(0, T ; V_{M}\right) \cap \mathcal{C}^{0}\left([0, T] ; L^{2}\left(Q_{M}\right)\right)$, with $\frac{\partial u_{M}}{\partial t} \in L^{2}\left(0, T ; V_{0, M}^{\prime}\right)$ such that, in the sense of the distributions in time,

$$
\begin{gathered}
\left\langle\frac{\partial \tilde{u}_{M}}{\partial t}, v\right\rangle+\left\langle\mathcal{L}_{t, 0} \tilde{u}_{M}, v\right\rangle=0 \quad \forall v \in V_{0, M}, \\
\tilde{u}_{M}(t, x, \pm M)=\left(K \mathrm{e}^{-r t}-x\right)_{+} \mathrm{e}^{-(1-\eta) \frac{(M-m)^{2}}{2 \nu^{2}}}
\end{gathered}
$$

and

$$
\tilde{u}_{M}(t=0, x, y)=u_{0}(x, y)
$$

It is possible to extend $\tilde{u}_{M}$ by $\left(K \mathrm{e}^{-r t}-x\right)_{+} \mathrm{e}^{-(1-\eta) \frac{(y-m)^{2}}{2 \nu^{2}}}$ out of $Q_{M}$ and the extension belongs to $L^{2}(0, T ; V) \cap$ $\mathcal{C}^{0}\left([0, T] ; L^{2}(Q)\right)$. We still call $\tilde{u}_{M}$ the extension. Under the same assumptions as in Theorem 1 , the sequence $\tilde{u}_{M}$ converges to the solution $u$ of $(28),(29)$ in $L^{2}(0, T ; V) \cap \mathcal{C}^{0}\left([0, T] ; L^{2}(Q)\right)$, as $M \rightarrow+\infty$. We call $\tilde{\chi}_{M}$ the function $\tilde{\chi}_{M}=\tilde{u}_{M}-\left(K \mathrm{e}^{-r t}-x\right) \mathrm{e}^{-(1-\eta) \frac{(y-m)^{2}}{2 \nu^{2}}}$. Here again, $\tilde{\chi}(t=0) \geq 0, \tilde{\chi}(t, x, \pm M) \geq 0$ and $\frac{\partial \tilde{\chi}}{\partial t}+\mathcal{L}_{t, 0} \tilde{\chi}=0$ in the sense of distributions. By reproducing the argument above, we conclude exactly as above that $\tilde{\chi} \geq 0$. By taking also $\mathrm{e}^{-\theta t} u_{-}$for $\theta \geq 0$ large enough, as a test-function in the equation satisfied by $u$, we obtain that $u \geq 0$. Therefore $u \geq\left(x-K \mathrm{e}^{-r t}\right)_{-} \mathrm{e}^{-(1-\eta) \frac{(y-m)^{2}}{2 \nu^{2}}}$ and the lower bound is obtained.

In the remaining part of Section 3.2, we shall prove additional regularity: we take formally the derivative with respect to $x$ of the equation satisfied by $P$, then we study the solution of this new problem, and deduce regularity for $P$.

Hereafter, we assume for simplicity that $\tilde{\gamma}$ does not depend on $x$. calling $R=\frac{\partial P}{\partial x}$, and taking formally the derivative with respect to $x$ of the equation satisfied by $P$, we obtain

$$
\frac{\partial R}{\partial t}+\frac{1}{2} y^{2} \frac{\partial}{\partial x}\left(x^{2} \frac{\partial R}{\partial x}\right)+\frac{1}{2} \beta^{2} \frac{\partial^{2} R}{\partial y^{2}}+r x \frac{\partial R}{\partial x}+(\alpha(m-y)-\beta \tilde{\gamma}(t, y)) \frac{\partial R}{\partial y}=0, \quad 0 \leq t<T, x>0, y \in \mathbb{R} .
$$

We call $w$ the function $w(t, x, y)=R(T-t, x, y) \mathrm{e}^{-(1-\eta) \frac{(y-m)^{2}}{2 \nu^{2}}}:$ we have

$$
\frac{\partial w}{\partial t}+\mathcal{H}_{t, 0} w=0
$$

where

$$
\begin{aligned}
\mathcal{H}_{t, 0}(w)= & -\frac{1}{2} y^{2} \frac{\partial}{\partial x}\left(x^{2} \frac{\partial w}{\partial x}\right)-\frac{1}{2} \beta^{2} \frac{\partial^{2} w}{\partial y^{2}}+(\beta \gamma(t, y)-(1-2 \eta) \alpha(y-m)) \frac{\partial w}{\partial y} \\
& -r x \frac{\partial w}{\partial x}+\left((1-\eta)\left(2 \frac{\alpha^{2}}{\beta^{2}} \eta(y-m)^{2}+2 \frac{\alpha}{\beta}(y-m) \gamma(t, y)\right)-\alpha(1-\eta)\right) w
\end{aligned}
$$

Proposition 4. Under the same assumptions as in Theorem 1, the equation (38), with the initial condition

$$
w(t=0, x, y)=-1_{\{x \leq K\}} \mathrm{e}^{-(1-\eta) \frac{(y-m)^{2}}{2 \nu^{2}}}
$$

has a unique weak solution in $L^{2}(0, T ; V) \cap \mathcal{C}^{0}\left([0, T] ; L^{2}(Q)\right)$, with $\frac{\partial w}{\partial t} \in L^{2}\left(0, T ; V^{\prime}\right)$.

Proof. The proof follows the same lines as that of Theorem 1. Concerning the partial differential operator, the term $-\frac{1}{2} y^{2} \frac{\partial}{\partial x}\left(x^{2} \frac{\partial w}{\partial x}\right)$ is in divergence form, so we have no additional restrictions on $\alpha$ and $\beta$. 
Also, the analogue of Theorem 2 can be applied, so if the condition (40) is replaced by $w(t=0)=w_{0}$ where $w_{0} \in D$ then the weak solution given by Proposition 4 belongs to $\mathcal{C}^{1}\left((0, T], L^{2}(Q)\right) \cap \mathcal{C}^{0}([0, T], D)$ (we have assumed that $\gamma$ is continuous in time).

Lemma 6. Under the same assumptions as in Proposition 4, there exists a unique $w_{M}$ in $L^{2}\left(0, T ; V_{0, M}\right) \cap$ $\mathcal{C}^{0}\left([0, T] ; L^{2}\left(Q_{M}\right)\right)$, with $\frac{\partial w_{M}}{\partial t} \in L^{2}\left(0, T ; V_{0, M}^{\prime}\right)$ defined as the weak solution of

$$
\frac{\partial w_{M}}{\partial t}+\mathcal{H}_{t, 0} w_{M}=0, \quad \text { in } \mathrm{Q}_{\mathrm{M}}
$$

with the initial condition

$$
w_{M}(t=0, x, y)=-1_{\{x \leq K\}} \mathrm{e}^{-(1-\eta) \frac{(y-m)^{2}}{2 \nu^{2}}}, \quad \text { in } \mathrm{Q}_{\mathrm{M}} .
$$

We extend $w_{M}$ by zero in $Q \backslash Q_{M}$ and we still call $w_{M}$ the extension.

Lemma 7. Under the same assumptions as in Proposition 4, the sequence $w_{M}$ converges to the weak solution $w$ of (38), (40) in $L^{2}(0, T ; V) \cap \mathcal{C}^{0}\left([0, T] ; L^{2}(Q)\right)$, as $M \rightarrow+\infty$.

We introduce the function $\Psi \in \mathcal{C}^{0}\left([0, T] ; L^{2}(-M, M)\right) \cap \mathcal{C}^{1}\left((0, T] ; L^{2}(-M, M)\right) \cap L^{2}\left(0, T ; H_{0}^{1}(-M, M)\right)$ as the weak solution of the parabolic problem

$$
\begin{aligned}
& \frac{\partial \Psi}{\partial t}-\frac{1}{2} \beta^{2} \frac{\partial^{2} \Psi}{\partial y^{2}}+(-\alpha(y-m)+\beta \gamma(t, y)) \frac{\partial \Psi}{\partial y}=0, \quad t \in(0, T), y \in(-M, M), \\
& \Psi(0, y)=K \quad y \in(-M, M) .
\end{aligned}
$$

Note that $\Psi \geq 0$ a.e.

By using the maximum principle, we can prove

Lemma 8. The function $u_{M}$ defined by (33), (34) satisfies

$$
\left(\Psi(t, y) \mathrm{e}^{-r t}-x\right)_{+} \mathrm{e}^{-(1-\eta) \frac{(y-m)^{2}}{2 \nu^{2}}} \leq u_{M}(t, x, y) \leq \Psi(t, y) \mathrm{e}^{-(1-\eta) \frac{(y-m)^{2}}{2 \nu^{2}}} \mathrm{e}^{-r t} .
$$

It can be checked that the function $\tilde{u}_{M}(t, x, y)=\Psi(t, y) \mathrm{e}^{-(1-\eta) \frac{(y-m)^{2}}{2 \nu^{2}}} \mathrm{e}^{-r t}+\int_{0}^{x} w_{M}(t, \xi, y) \mathrm{d} \xi$ is such that for all $\epsilon>0, \quad \tilde{u}_{M} \mathrm{e}^{-\epsilon \log ^{2}(x)} \in L^{2}\left(0, T ; V_{0, M}\right) \cap \mathcal{C}^{0}\left([0, T] ; L^{2}\left(Q_{M}\right)\right)$ and that $\frac{\partial}{\partial t} \tilde{u}_{M} \mathrm{e}^{-\epsilon \log ^{2}(x)} \in L^{2}\left(0, T ; V_{0, M}^{\prime}\right)$. Moreover, for any $\phi \in \mathcal{C}^{\infty}\left([0, T] \times Q_{M}\right)$, with compact support in $(x, y)$,

$$
\left\langle\frac{\partial \tilde{u}_{M}}{\partial t}+\mathcal{L}_{t, 0} \tilde{u}_{M}, \frac{\partial \phi}{\partial x}\right\rangle=0
$$

This proves that $\frac{\partial \tilde{u}_{M}}{\partial t}+\mathcal{L}_{t, 0} \tilde{u}_{M}=g(t, y)$. Furthermore

$$
\tilde{u}_{M}(t=0)=u_{M}(t=0),
$$

and

$$
\tilde{u}_{M}(t, x, M)=\tilde{u}_{M}(t, x,-M)=0 .
$$


Again, by using the maximum principle, it is possible to prove that $\tilde{u}_{M}=u_{M}+f(t, y)$, where

$$
\begin{aligned}
& \frac{\partial f}{\partial t}-\frac{1}{2} \beta^{2} \frac{\partial^{2} f}{\partial y^{2}}+(-(1-2 \eta) \alpha(y-m)+\beta(\gamma(t, y))) \frac{\partial f}{\partial y} \\
& +\left(r+2 \frac{\alpha^{2}}{\beta^{2}} \eta(1-\eta)(y-m)^{2}+2(1-\eta) \frac{\alpha}{\beta}(y-m) \gamma(t, y)-\alpha(1-\eta)\right) f=g(t, y), \\
& f(0, y)=0 \quad y \in(-M, M), \\
& f(t, \pm M)=0 .
\end{aligned}
$$

Also, from (44), we have $f=0$. We have proved:

Lemma 9. The functions $u_{M}$ and $w_{M}$ defined above satisfy

$$
u_{M}(t, x, y)=\Psi(t, y) \mathrm{e}^{-(1-\eta) \frac{(y-m)^{2}}{2 \nu^{2}}} \mathrm{e}^{-r t}+\int_{0}^{x} w_{M}(t, \xi, y) \mathrm{d} \xi
$$

Lemma 10. The functions $u$ and $w$ defined respectively by (28), (29) and (38), (40) satisfy

$$
u(t, x, y)=K \mathrm{e}^{-(1-\eta) \frac{(y-m)^{2}}{2 \nu^{2}}} \mathrm{e}^{-r t}+\int_{0}^{x} w(t, \xi, y) \mathrm{d} \xi .
$$

Proof. From Lemmas 4 and 7, we can pass to the limit in (47).

Finally we have proved that:

Theorem 3. Under the same assumptions as in Theorem 2 and if $\gamma$ does not depend on $x$, the solution of (28), (29) belongs to $\mathcal{C}^{0}\left(0, T ; W_{1}\right)$ and to $L^{2}\left(0, T ; W_{2}\right)$, where $W_{1}=H^{1}(Q)$ and $W_{2}=\left\{w \in V, \frac{\partial w}{\partial x} \in V\right\}$.

Remark 8. It is not possible to iterate the arguments above to prove a stronger global regularity result, because the second derivative of the initial condition with respect to $x$ is not a square integrable function. Indeed, the initial condition is not regular near the strike.

Remark 9. As a consequence of Theorem 3, it is possible to use standard finite elements to approximate $u$.

Remark 10. If $\gamma$ is smooth enough, it is also possible to prove better local regularity results away from the strike. In particular, these results show that, when using finite difference or finite element methods, it is not necessary to refine the mesh near $x=0$, as it is commonly done in the quantitative finance codes.

\subsection{The correlated case}

We consider now the case when $\rho \neq 0$.

Proposition 5. Assume that $r$ is a bounded function of time and that $\gamma$ is bounded by a constant. Assume that the ratio $\omega=\frac{\alpha}{\beta}$ satisfies

$$
\omega^{4}-\frac{3}{2}|\rho| \omega^{3}-\omega^{2}+\frac{9}{8} \rho^{2} \omega-\frac{9}{16} \rho^{2}>0
$$

and that

$$
\beta>4 \frac{|\rho|}{1-|\rho|} \sup _{t}|\mu-r(t)|,
$$

then there exist two positive constants $C$ and $c$ independent on $t$ and two constants $0<\eta_{1}<\eta_{2}<1$ such that, for $\eta_{1}<\eta<\eta_{2}$ and for any $v \in V_{0, \pm}$, where the space $V_{0, \pm}$ is defined in Section 3.3

$$
\left\langle\mathcal{L}_{t, \rho} v, v\right\rangle \geq C\|v\|_{V_{0, \pm}}^{2}-c\|v\|_{L^{2}\left(Q_{ \pm}\right)}^{2} .
$$


Proof. We choose $v \in V_{0,+}$. The arguments are identical for $v \in V_{0,-}$. We can write

$$
\left\langle\mathcal{L}_{t, \rho} v, v\right\rangle=\sum_{i=1}^{10} \mathcal{I}_{i}
$$

where

$$
\begin{gathered}
\mathcal{I}_{1}=\frac{1}{2}\left\|x y \frac{\partial v}{\partial x}\right\|_{L^{2}\left(Q_{+}\right)}^{2}-\frac{1}{2}\|y v\|_{L^{2}\left(Q_{+}\right)}^{2}, \quad \mathcal{I}_{2}=\frac{\beta^{2}}{2}\left\|\frac{\partial v}{\partial y}\right\|_{L^{2}\left(Q_{+}\right)}^{2}, \quad \mathcal{I}_{3}=\frac{r(t)}{2}\|v\|_{L^{2}\left(Q_{+}\right)}^{2}, \\
\mathcal{I}_{4}=\int_{Q_{+}}\left(-\alpha(1-2 \eta)(y-m)+\sqrt{1-\rho^{2}} \beta \gamma(t, x, y)\right) v \frac{\partial v}{\partial y}, \\
\mathcal{I}_{5}=\int_{Q_{+}}\left(r(t)+2(1-\eta) \frac{\alpha}{\beta}(y-m) \sqrt{1-\rho^{2}} \gamma(t, x, y)-\alpha(1-\eta)\right) v^{2}, \\
\mathcal{I}_{6}=2 \frac{\alpha^{2}}{\beta^{2}} \eta(1-\eta) \int_{Q_{+}}(y-m)^{2} v^{2}, \\
\mathcal{I}_{7}=-\rho \beta\left\langle x y \frac{\partial^{2} v}{\partial x \partial y}, v\right\rangle, \quad \mathcal{I}_{8}=2 \rho \frac{\alpha}{\beta}(1-\eta) \int_{Q_{+}}(m-y) x y v \frac{\partial v}{\partial x}, \\
\mathcal{I}_{9}=\rho \beta \int_{Q_{+}} \frac{\mu-r(t)}{y} v \frac{\partial v}{\partial y}, \quad \mathcal{I}_{10}=2 \rho(1-\eta) \frac{\alpha}{\beta} \int_{Q_{+}}(y-m) \frac{\mu-r(t)}{y} v^{2} .
\end{gathered}
$$

The quantities $\mathcal{I}_{1}-\mathcal{I}_{6}$ are bounded from below exactly as in the proof of Proposition 2, and

$$
\begin{gathered}
\mathcal{I}_{7}=\rho \beta \int_{Q_{+}} y x \frac{\partial v}{\partial y} \frac{\partial v}{\partial x}-\frac{\rho \beta}{2} \int_{Q_{+}} v^{2} \geq-|\rho| \frac{\beta^{2}}{2}\left\|\frac{\partial v}{\partial y}\right\|_{L^{2}\left(Q_{+}\right)}^{2}-\frac{|\rho|}{2}\left\|x y \frac{\partial v}{\partial x}\right\|_{L^{2}\left(Q_{+}\right)}^{2}-\frac{|\rho| \beta}{2}\|v\|_{L^{2}\left(Q_{+}\right)}^{2}, \\
\mathcal{I}_{8}=\rho \frac{\alpha}{\beta}(1-\eta) \int_{Q_{+}}|y|(y-m) v^{2} \geq-\frac{3}{2}|\rho| \frac{\alpha}{\beta}(1-\eta)\|y v\|_{L^{2}\left(Q_{+}\right)}^{2}-|\rho| m^{2} \frac{\alpha}{\beta}(1-\eta)\|v\|_{L^{2}\left(Q_{+}\right)}^{2} .
\end{gathered}
$$

By the Hardy's inequality,

$$
\begin{gathered}
\mathcal{I}_{9}=\frac{\rho \beta}{2}(\mu-r(t)) \int_{Q_{+}}\left(\frac{v}{y}\right)^{2} \geq-2|\rho| \beta|\mu-r(t)|\left\|\frac{\partial v}{\partial y}\right\|_{L^{2}\left(Q_{+}\right)}^{2}, \\
\mathcal{I}_{10} \geq-2|\rho|(1-\eta) \frac{\alpha}{\beta}|\mu-r(t)|\left(\left(1+\frac{|m| \zeta}{2}\right)\|v\|_{L^{2}\left(Q_{+}\right)}^{2}+2 \frac{|m|}{\zeta}\left\|\frac{\partial v}{\partial y}\right\|_{L^{2}\left(Q_{+}\right)}^{2}\right),
\end{gathered}
$$

by assembling the estimates above, we obtain an equality of the type

$$
a_{1}\left\|x y \frac{\partial v}{\partial x}\right\|_{L^{2}\left(Q_{+}\right)}^{2}+a_{2}\left\|\frac{\partial v}{\partial y}\right\|_{L^{2}\left(Q_{+}\right)}^{2}+a_{3}\|y v\|_{L^{2}\left(Q_{+}\right)}^{2}+a_{4}\|v\|_{L^{2}\left(Q_{+}\right)}^{2} \geq 0 .
$$

Clearly $a_{1}=\frac{1}{2}(1-\rho)>0$. We have to bound from below the coefficients $a_{2}$ and $a_{3}$. The condition (49) says that $\max _{\eta} \lim _{\zeta \rightarrow \infty} a_{3}>0$ and the condition (50) says that $\lim _{\zeta \rightarrow \infty} a_{2}>0$. If the conditions (49), (50) are satisfied, it is possible to choose $C, 0<C<\frac{1}{2}$ and $\eta_{1}, \eta_{2}, 0<\eta_{1}<\eta_{2}<1$, such that, for any $\eta$ with $\eta_{1}<\eta<\eta_{2}$ and for $\zeta$ large enough,

$$
a_{3}=-\frac{1}{2}+(1-\eta) \frac{\alpha}{\beta}\left(2 \frac{\alpha}{\beta} \eta\left(1-\frac{|m|}{\zeta}\right)-\frac{3}{2}|\rho|-\sqrt{1-\rho^{2}} \frac{\Gamma}{\zeta}\right)>C
$$


and at the same time,

$$
a_{2}=\frac{\beta}{2}\left((1-|\rho|) \beta-\sqrt{1-\rho^{2}} \frac{\Gamma}{\zeta}-4|\rho||\mu-r(t)|\right)-4|\rho|(1-\eta) \frac{\alpha}{\beta} \frac{|m|}{\zeta}|\mu-r(t)|>C .
$$

Then the estimate (51) follows by fixing $\zeta$.

Remark 11. Here again, the assumption on the ratio $\frac{\alpha}{\beta}$ is usually satisfied in practice. It can be slightly relaxed by using (19).

Consider a European vanilla put, whose payoff function is $h(x, y)=(x-K)_{-}$. We have seen that if $\rho \neq 0$, we have to impose the boundary condition $P(t, x, 0)=\left(x-K \mathrm{e}^{-\int_{t}^{T} \hat{r}(\xi) d \xi}\right)_{-}$, which is the solution of (12) if $h(x)=(x-K)_{-}$. After the change of unknown (13), the initial condition for $u$ is (27). Let us call

$$
\bar{u}(t, x, y)=\mathrm{e}^{-(1-\eta) \frac{(y-m)^{2}}{2 \nu^{2}}}\left(x-K \mathrm{e}^{-\int_{0}^{t} r(\xi) d \xi}\right)_{-} .
$$

\section{Proposition 6.}

$$
\frac{\partial \bar{u}}{\partial t}+\mathcal{L}_{t, \rho} \bar{u} \in L^{2}\left(0, T ; V_{0, \pm}^{\prime}\right)
$$

Proof. The proof is similar as that of Proposition 1.

Theorem 4. If the assumptions of Proposition 5 are satisfied, and if $\eta$ is chosen as in Proposition 5, there exists a unique $u^{ \pm}$in $L^{2}\left(0, T ; V_{0, \pm}\right) \cap \mathcal{C}^{0}\left([0, T] ; L^{2}\left(Q_{ \pm}\right)\right)$, with $\frac{\partial u^{ \pm}}{\partial t} \in L^{2}\left(0, T ; V_{0, \pm}^{\prime}\right)$ such that, in the sense of the distributions,

$$
\frac{\partial u^{ \pm}}{\partial t}+\mathcal{L}_{t, \rho} u^{ \pm}=-\frac{\partial \bar{u}}{\partial t}-\mathcal{L}_{t, \rho} \bar{u}
$$

and

$$
u^{ \pm}(t=0)=0
$$

Proof. The proof stems from the general results of [9].

Call $u$ the function obtained by gluing together the functions $u^{ \pm}$and by adding to the result the function $\bar{u}$. The function $P(t, x, y)=u(T-t, x, y) \mathrm{e}^{(1-\eta) \frac{(y-m)^{2}}{2 \nu^{2}}}$ satisfies $(5)$ in $Q_{+}$and $Q_{-}$.

Proposition 7. Under the assumption of Theorem 4, and if $r$ is constant in time and $\gamma$ is continuous in time, the function $P$ satisfies

$$
\left(x-K \mathrm{e}^{-r(T-t)}\right)_{-} \leq P(t, x, y) \leq K \mathrm{e}^{-r(T-t)} .
$$

Proof. The proof follows the same lines as that of Proposition 3.

\section{Generalization}

It is possible to generalize all the results above and carry out a variational analysis for the equation (5) where $f$ is an arbitrary function such that:

1. $f \in W^{2, \infty}(\mathbb{R})$, and in particular we assume that $\left|f^{\prime \prime}\right| \leq M$ a.e.

2. The zeros of $f$ are simple and there is a finite number of zeros. 
3. There exists a constant $c$ such that

$$
\max \left(|f(y)|,\left|f^{\prime}(y)\right|\right) \geq c .
$$

We shall not reproduce all the arguments above: instead, we shall focus on the case where $\rho=0$ and only give details for the proof of the analogue of Lemma 2. All the other results are proved exactly in the same way as for $f(y)=|y|$. When $\rho \neq 0$, the analysis mimics exactly what has been presented above: one has to introduce Dirichlet conditions where $f(y)$ vanishes, and use the Hardy inequality.

So let us take $\rho=0$ and consider the weighted Sobolev space $V$ :

$$
V=\left\{v:\left(\sqrt{y^{2}+1} v, \frac{\partial v}{\partial y}, x f(y) \frac{\partial v}{\partial x}\right) \in\left(L^{2}(Q)\right)^{3}\right\}
$$

This space with the norm

$$
\|v\|_{V}=\left(\int_{Q}\left(y^{2}+1\right) v^{2}+\left(\frac{\partial v}{\partial y}\right)^{2}+x^{2} f(y)^{2}\left(\frac{\partial v}{\partial x}\right)^{2}\right)^{\frac{1}{2}}
$$

is a Hilbert space, and it has the following properties:

1. $V$ is separable and reflexive. We call $V^{\prime}$ the dual of $V$.

2. $\mathcal{D}(Q) \subset V$ and $\mathcal{D}(Q)$ is dense in $V$, (by using a result of Franchi et al. [6]).

3. $V$ is dense in $L^{2}(Q)$.

We look for the weak solution of the degenerate parabolic equation

$$
\begin{aligned}
& \frac{\partial u}{\partial t}+\mathcal{L}_{t, 0} u=0, \\
& u(t=0)=(x-K) \_\mathrm{e}^{(1-\eta) \frac{(y-m)^{2}}{2 \nu^{2}}}
\end{aligned}
$$

where

$$
\begin{aligned}
\mathcal{L}_{t, 0}(v)=-\frac{1}{2} f(y)^{2} x^{2} \frac{\partial^{2} v}{\partial x^{2}}-\frac{1}{2} \beta^{2} \frac{\partial^{2} v}{\partial y^{2}}-r(t) x \frac{\partial v}{\partial x}+(-(1-2 \eta) \alpha(y-m)+\beta \gamma(t, x, y)) \frac{\partial v}{\partial y} \\
+\left(r(t)+2 \frac{\alpha^{2}}{\beta^{2}} \eta(1-\eta)(y-m)^{2}+2(1-\eta) \frac{\alpha}{\beta}(y-m) \gamma(t, x, y)-\alpha(1-\eta)\right) v .
\end{aligned}
$$

We have the estimate:

Lemma 11. Let $v$ be a function in $V$. Then

$$
\int_{Q} f(y)^{2} v^{2} \leq 4 \int_{Q} f(y)^{2} x^{2}\left(\frac{\partial v}{\partial x}\right)^{2}
$$

Also,

Lemma 12. The operator $v \rightarrow \beta x \frac{\partial v}{\partial x}$ is continuous from $V$ into $V^{\prime}$.

Proof. Call $X$ and $Y$ the differential operators

$$
X(v)=x f(y) \frac{\partial v}{\partial x}+\beta \frac{\partial v}{\partial y}, \quad Y(v)=x f(y) \frac{\partial v}{\partial x}-\beta \frac{\partial v}{\partial y},
$$


The operators $X$ and $Y$ are continuous operators from $V$ into $L^{2}(Q)$ and their adjoints are

$$
\begin{aligned}
X^{T}(v) & =-x f(y) \frac{\partial v}{\partial x}-\beta \frac{\partial v}{\partial y}-f(y) v=-X(v)-f(y) v, \\
Y^{T} v & =-x f(y) \frac{\partial v}{\partial x}+\beta \frac{\partial v}{\partial y}-f(y) v=-Y(v)-f(y) v .
\end{aligned}
$$

Consider the commutator $[X, Y]=X Y-Y X$ : it can be checked that

$$
[X, Y](v)=2 \beta x f^{\prime}(y) \frac{\partial v}{\partial x} .
$$

Suppose for simplicity that $f$ has only one zero $y_{0}$. When the number of zeros is not one, the modifications to the proof are easy. There exists three positive constants $c c^{\prime}$ and $\xi$ such that $\left|f^{\prime}(y)\right|>c$ and $|f(y)| \geq c\left|y-y_{0}\right|$ if $\left|y-y_{0}\right|<\xi$ and $f(y)>c^{\prime}$ if $\left|y-y_{0}\right| \geq \frac{\xi}{2}$. Let $\psi$ be a smooth function such that:

1. $0 \leq \psi \leq 1$,

2. $\psi(y)=1$ if $\left|y-y_{0}\right| \leq \frac{\xi}{2}$,

3. $\psi(y)=0$ if $\left|y-y_{0}\right| \geq \xi$.

For $v \in V$ and $w \in \mathcal{D}(Q)$,

$$
\begin{aligned}
& \left|c\left\langle 2 \beta x \frac{\partial v}{\partial x}, \psi(y) w\right\rangle\right|=\frac{c}{\left|f^{\prime}\left(y_{0}\right)\right|}\left|\left\langle 2 \beta f^{\prime}\left(y_{0}\right) x \frac{\partial v}{\partial x}, \psi(y) w\right\rangle\right| \\
& \leq\left|\left\langle 2 \beta f^{\prime}\left(y_{0}\right) x \frac{\partial v}{\partial x}, \psi(y) w\right\rangle\right| \\
& \leq\left|\left\langle 2 \beta f^{\prime}(y) x \frac{\partial v}{\partial x}, \psi(y) w\right\rangle\right|+2|\beta| M \int\left|y-y_{0}\right| \psi(y)\left|x \frac{\partial v}{\partial x}\right||w| \\
& \leq\left|\left\langle 2 \beta f^{\prime}(y) x \frac{\partial v}{\partial x}, \psi(y) w\right\rangle\right|+2 \beta \frac{M}{c} \int|f(y)| \psi(y)\left|x \frac{\partial v}{\partial x}\right||w| .
\end{aligned}
$$

But

$$
\left|\left\langle 2 \beta f^{\prime}(y) x \frac{\partial v}{\partial x}, \psi(y) w\right\rangle\right|=\left|-\int_{Q} Y(v)(X(\psi(y) w)+f(y) \psi(y) w)+\int_{Q} X(v)(Y(\psi(y) w)+f(y) \psi(y) w)\right|,
$$

and

$$
\int|f(y)| \psi(y)\left|x \frac{\partial v}{\partial x}\right||w| \leq\|v\|_{V}\|w\|_{L^{2}(Q)}
$$

and from (59), there exists a constant $C$ such that

$$
\left|\left\langle 2 \beta x \frac{\partial v}{\partial x}, \psi(y) w\right\rangle\right| \leq C\|v\|_{V}\|w\|_{V} .
$$

Also

$$
\left|\left\langle 2 \beta x \frac{\partial v}{\partial x},(1-\psi(y)) w\right\rangle\right| \leq \frac{1}{c^{\prime}}\left\langle 2 \beta|f(y)|\left|x \frac{\partial v}{\partial x}\right|,(1-\psi(y))|w|\right\rangle \leq C\|v\|_{V}\|w\|_{V} .
$$

To conclude, we add the previous two estimates and we use the density of $\mathcal{D}(Q)$ into $V$.

With Lemma 12, it is possible to prove:

Proposition 8. Assume that $r$ is a bounded function of time and that $\gamma$ is bounded by a constant. The operator $\mathcal{L}_{t, 0}$ is a bounded linear operator from $V$ into $V^{\prime}$ with a constant independent of $t$. 
Thanks to Lemma 12, we can prove also the analogue of Theorem 1 for the case $\rho=0$, with the same assumption, namely $\alpha>\beta$.

\section{Numerical SOLUTiON}

\subsection{Description of the method}

All the computations below are done with $f(y)=|y|$ and $\tilde{\gamma}=0$.

\subsection{An uncorrelated case}

We want to price a European put with the following parameters

$$
r=0.05, \quad \rho=0, \quad \alpha=1, \quad \nu=0.5, \quad m=0.2 \quad K=20, \quad T=1 \text { year. }
$$

Remark 12. The choice of $\alpha=1$ is not quite realistic from a financial viewpoint, if the asset is linked to actions, because the mean reversion rate is generally larger. When the asset corresponds to interest rates, smaller values of $\alpha$ are reasonable. Larger values of $\alpha$ will be addressed below. When the mean reversion rate is large, it is possible to carry out an asymptotic expansion of the solution as in [5] and we believe that the variational setting introduced above permits to justify fully these expansions. This will be the topic of a forthcoming work. For smaller mean reversion rates, such expansions cannot be done, and the numerical computations are useful to understand the qualitative properties of the solutions.

The aim is to approximate $P$ in the domain $(0, \bar{x}) \times(-1.5,1.5)$ for $t$ smaller than 1 . We choose $\bar{x}=600$. For computing the solution, we discretize the equation (5) in the larger domain $(0,600) \times(-4,4)$. We use artificial Dirichlet conditions on the boundaries $y= \pm 4$. These conditions, which are obviously not satisfied by $P$, induce nevertheless small errors on $P$ in the smaller domain $(0,600) \times(-1.5,1.5)$ because the advection terms are strong near the top and bottom boundaries, and directed outward, so the artificial boundary conditions just produce a boundary layer. Indeed, the advection term in the $y$-direction, namely $\alpha(y-m) \frac{\partial P}{\partial y}$ dominates very much the diffusion term $-\frac{\beta^{2}}{2} \frac{\partial^{2} P}{\partial y^{2}}$, except in a small boundary layer, because for $y= \pm 4$ the ratio $\frac{2 \alpha(y-m)}{\beta^{2}}=\frac{y-m}{\nu^{2}} \sim 16$.

On the boundary $x=\bar{x}$, we impose $\frac{\partial u}{\partial x}(\bar{x})=0$. No boundary condition is needed on $x=0$, because of the degeneracy of the equation.

We have used two methods in order to discretize the problem:

1. A piecewise linear conforming finite element method on an unstructured triangular mesh. The time scheme is a first order implicit Euler scheme and the advection terms (first order partial derivatives in $x, y$ ) are discretized by the method of the characteristics, see [4]. One important advantage of the method of characteristics is that it is unconditionally stable in the $L^{2}$ norm. The computations were done by using the free software FreeFem from O. Pironneau and F. Hecht $[11,12]$.

The time step used in these calculations is one day. The mesh is refined adaptively every ten days and the refinement is done according to the metric generated by the Hessian of the computed solution, see [12]. The method is first order accurate in time and second order accurate in the $x, y$ variables. It is possible to improve the method of characteristics to have also second order accuracy in time, but it has been done here.

2. A finite difference method on a uniform mesh. The time scheme is a second order Crank-Nicolson scheme, and the first order partial derivatives in $x, y$ are discretized by a first order upwind scheme. Upwinding is very important because when $|y|$ is large, the equation is advection-dominated. A centered scheme yields blow-up of the solution. The method is second order accurate in time and only first order accurate in $x, y$ because of upwinding.

The time step was set to 3.33 days, the $x$-step was 1 , and the $y$-step was 0.1 .

The large sparse linear systems arising in the scheme are solved iteratively by means of a bicg-stab method [15]. 

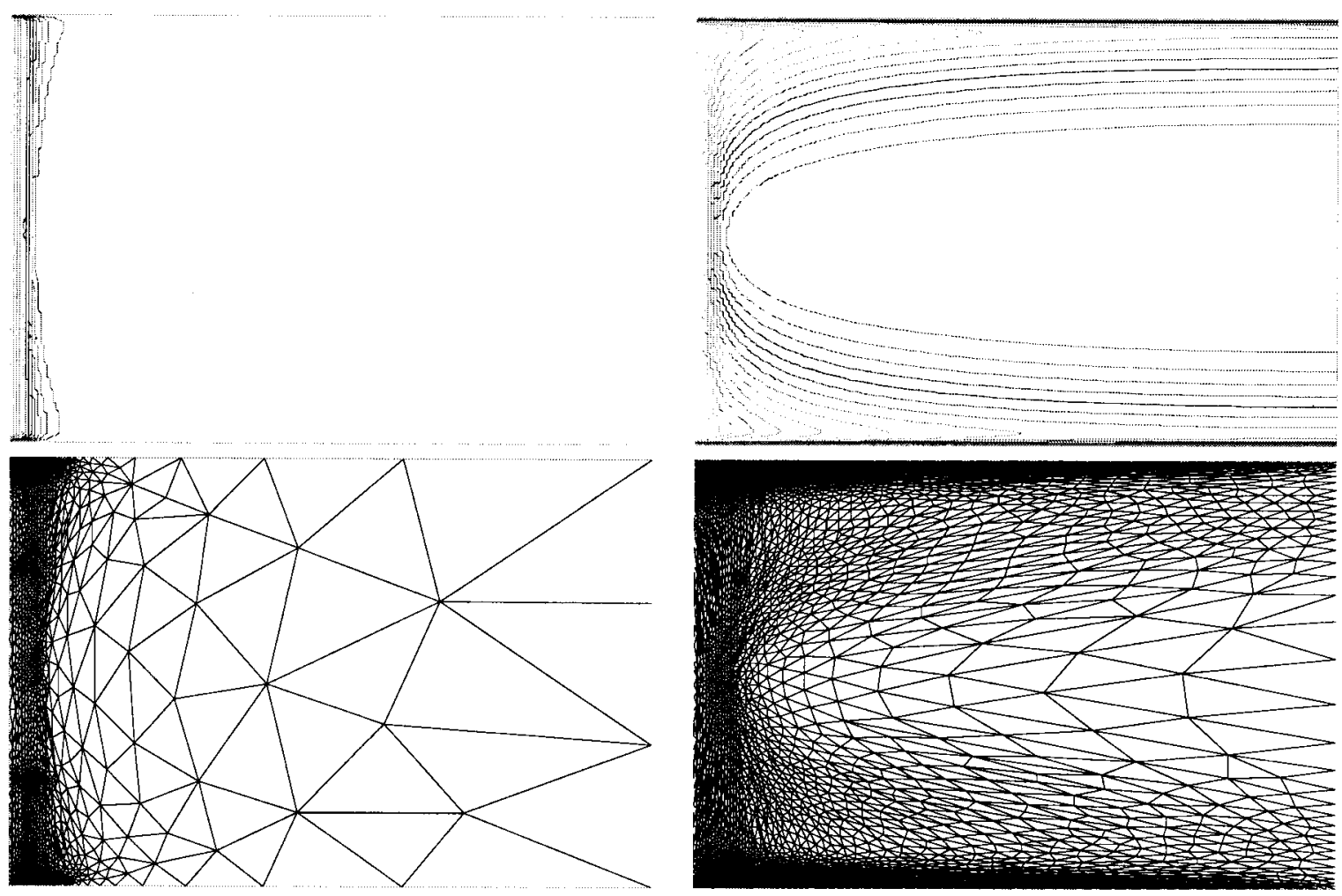

Figure 1 . The price of the put for $x \in(0,600), y \in(-4,4)$, computed by the finite element method. The values of $y$ greater than one are useless from a financial point of view, but since we use an artificial boundary condition, the boundary of the computational domain i.e. $y= \pm 4$ needs to be far enough from the zone of practical interest. The solution has been successively represented at ten days before the expiration date, and at $t=0$. Note that the effect of the artificial boundary conditions is limited to boundary layers. On the bottom, the adapted mesh at these two dates.

Remark 13. A priori error estimates for finite elements for the rather similar degenerate operator $\frac{\partial^{2}}{\partial y^{2}}+y^{2} \frac{\partial^{2}}{\partial x^{2}}$ are available in [7]. In this paper, Franchi et al. prove that if the mesh points are distributed uniformly in $x\left(x_{i}=\frac{i}{n}\right)$ and with a special law in the $y$-direction $\left(y_{i}=\sqrt{\frac{i}{n}}\right)$ then the error in the operator-norm decays like $\frac{1}{\sqrt{n}}$. In our case, we have $\frac{1}{2} x^{2} y^{2} \frac{\partial^{2}}{\partial x^{2}}$ instead of $y^{2} \frac{\partial^{2}}{\partial x^{2}}$, but we believe that in the case of interest, this additional degeneracy will not modify the estimates of [7], because the solution is very regular near $x=0$. Of course, in order to derive estimates for the problem described in this paper, one has also to take into account all the lower order partial differential operators in (5). This will done in a forthcoming work.

In Figure 1, we show the solutions obtained with the finite element method at different times. In Figure 2, we show the same solution obtained with the finite difference method. The difference between the computed solutions is not visible, but it is clear that mesh adaption in the finite element method permits a greater accuracy. In Figure 3, we show the solutions obtained at different times where all the parameters except $\alpha$ are unchanged and $\alpha=250$ : here we have used the finite element method described above. The solution has a very little dependency on $y$. As in [5] it should be possible to carry out an asymptotic expansion for large values of $\alpha$ and to find that up to leading order, the solution satisfies an equation independent on $y$. 

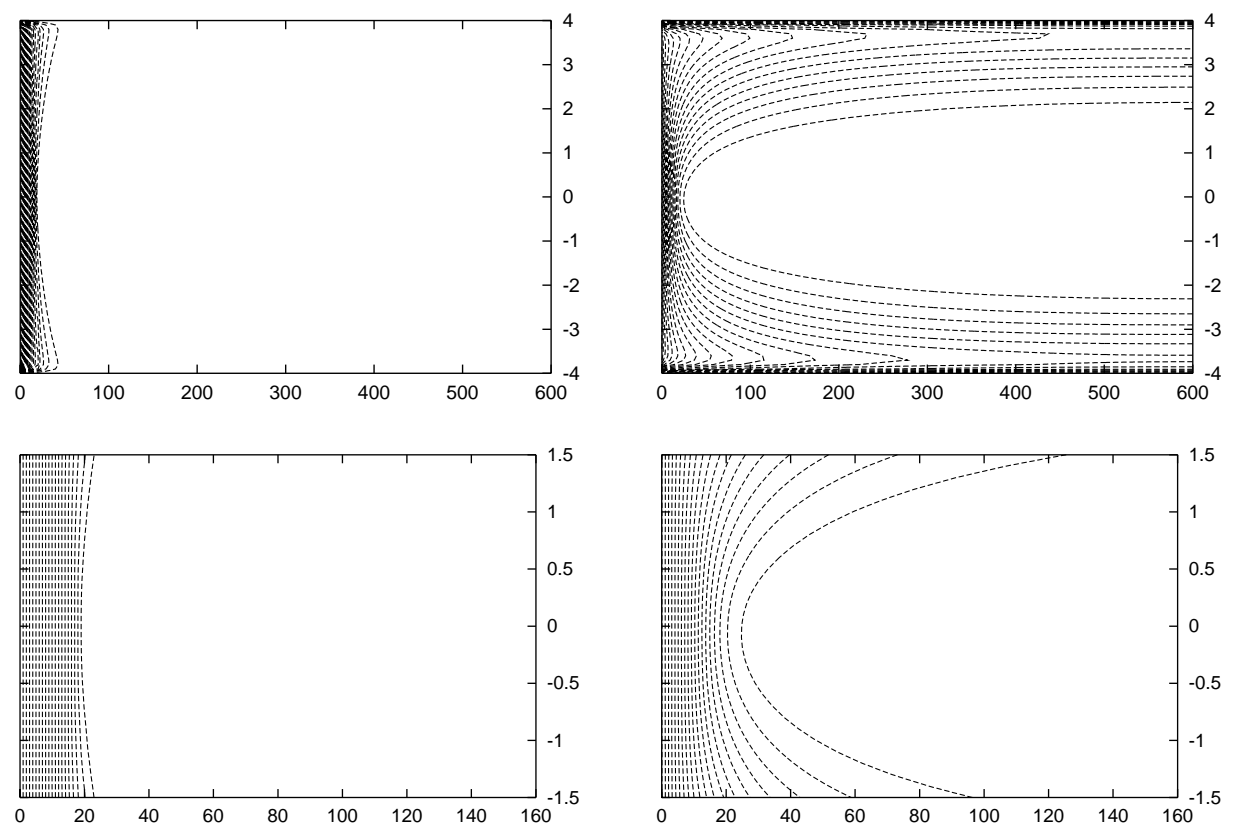

Figure 2. On top, the price of the put for $x \in(0,600), y \in(-4,4)$ computed by a Crank Nicolson scheme with an upwinded finite difference method. The solution is represented at ten days before the expiration date, and at $t=0$. These results are in good agreement with those obtained by the finite element method. The finite element results are more accurate because the mesh is adaptively refined. Also, thanks to adaptive refinment, the simulation based on finite elements takes less than a tenth of the computing time needed by the finite difference scheme. On the bottom, zooms in the region $x \in(0,150), y \in(-1.5,1.5)$. Note that the solution is not symmetric in the variable $y$ because $m \neq 0$.
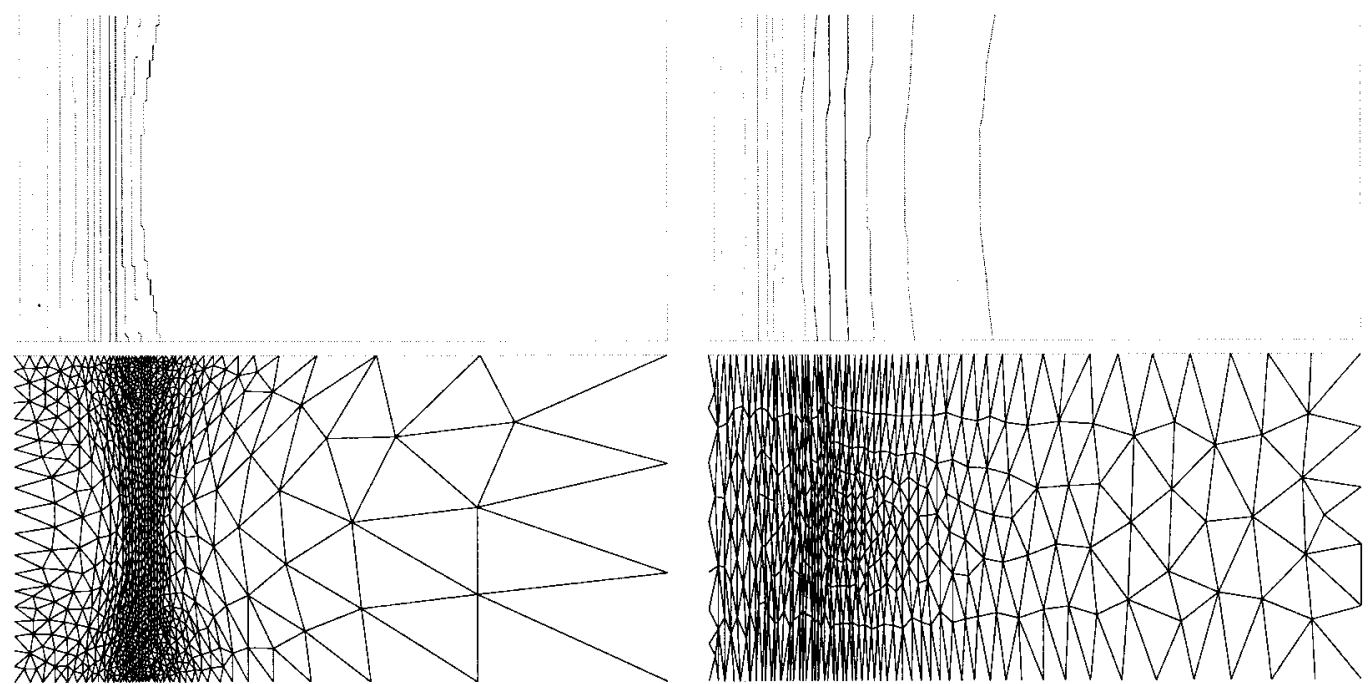

Figure 3. The price of the put for $y \in(-4,4)$ and $x \in(0,100)$. The solution has been successively represented ten days before the expiration date, and at $t=0$. On the bottom, the adapted meshes at these dates. 

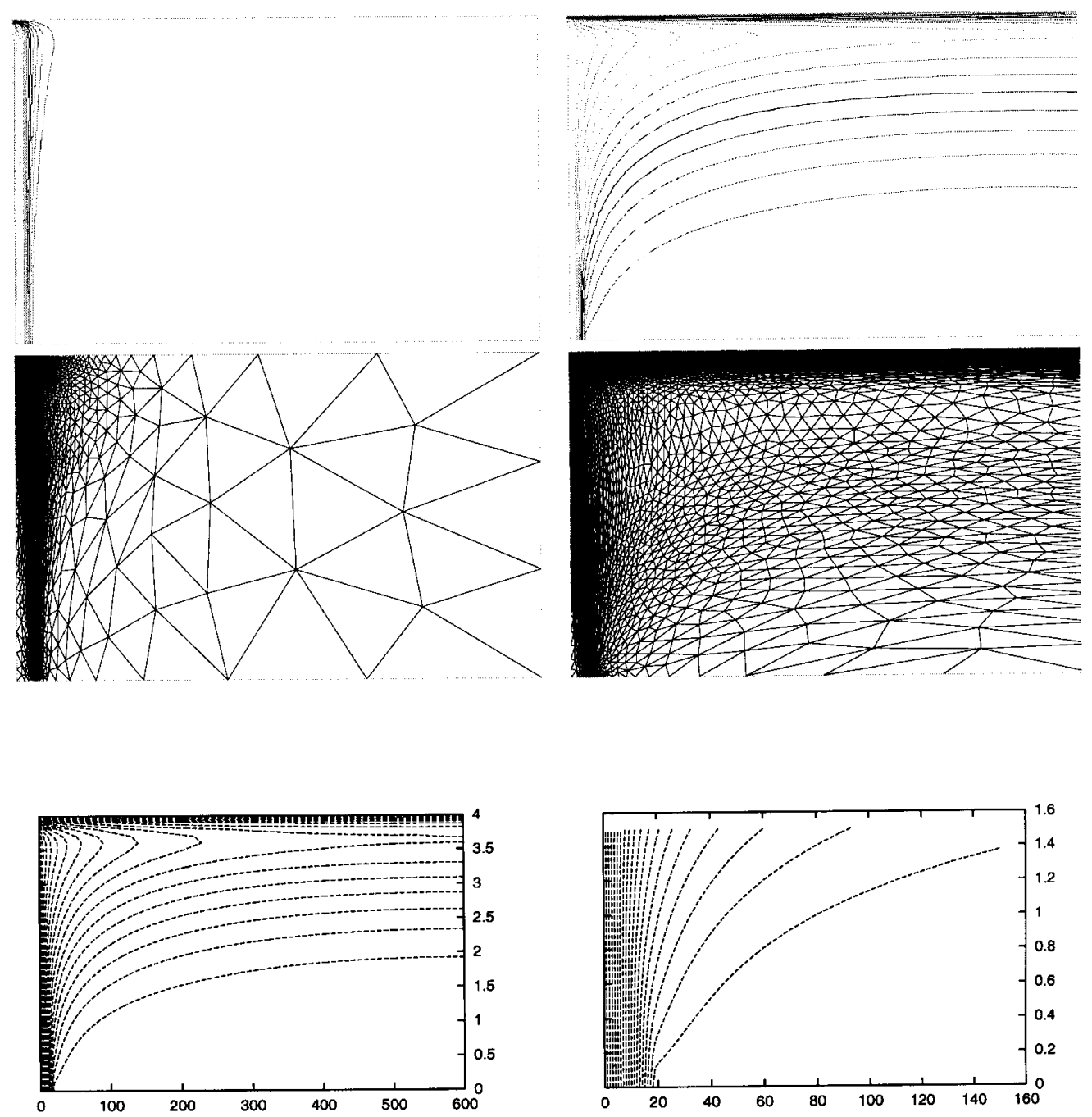

Figure 4. The price of the put for $x \in(0,600), y \in(0,4)$. On top, the solution computed by a finite element method has been successively represented at 10 days before the expiration date, and at $t=0$. The solution is not correct in the upper part in the domain but the error is restricted to a boundary layer. In the center, the mesh is adapted during the time stepping scheme. On the bottom, the price $P$ computed by a finite difference method one year before the expiration date and a zoom for $x \in(0,150), y \in(0,1.5)$. The two computations are in good agreement.

\subsection{A correlated case}

We consider the price of a European put with the following parameters

$$
r=0.05, \quad \rho=-0.5, \quad \alpha=1, \quad \nu=0.5, \quad m=0.2 \quad \mu=0.15, \quad K=20, \quad T=1 \text { year. }
$$

The function $\gamma$ is taken to be zero.

In Figure 4, we show the solutions obtained at different times for positive values of $y$. As above, they are computed by using a finite element method with adapted meshes. 

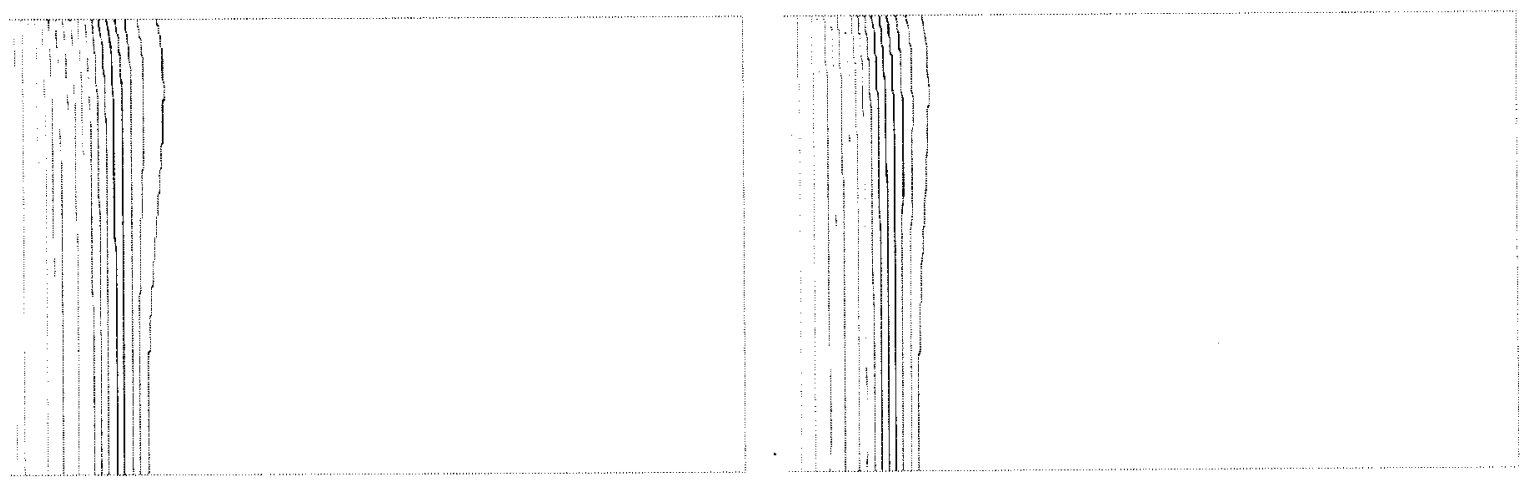

Figure 5. The price of the put for $x \in(0,100)$ and $y \in(0,1.5)$. The solution has been successively represented at: ten days before the expiration date, and at $t=0$.

The solution is computed in the domain $(0,600) \times(0,4)$.

In Figure 5, we show the solutions obtained at different times where all the parameters except $\alpha$ are unchanged and $\alpha=250$. The solution varies very little with respect to time.

Acknowledgements. It is a pleasure to thank Bruno Franchi for helpful discussions.

\section{REFERENCES}

[1] Y. Achdou and B. Franchi (in preparation).

[2] H. Brezis, Analyse Fonctionnelle, Théorie et Applications. Masson (1983).

[3] T. Cazenave and A. Haraux, An introduction to semilinear evolution equations. The Clarendon Press Oxford University Press, New York (1998). Translated from the 1990 French original by Y. Martel and revised by the authors.

[4] J. Douglas and T.F. Russell, Numerical methods for convection dominated diffusion problems based on combining the method of characteristics with finite element methods or finite difference method. SIAM J. Numer. Anal. 19 (1982) 871-885.

[5] J.-P. Fouque, G. Papanicolaou and K. Ronnie Sircar. Derivatives in financial markets with stochastic volatility. Cambridge University Press, Cambridge (2000).

[6] B. Franchi, R. Serapioni and F. Serra Cassano. Meyers-Serrin type theorems and relaxation of variational integrals depending on vector fields. Houston J. Math. 22 (1996) 859-890.

[7] B. Franchi and M.C. Tesi, A finite element approximation for a class of degenerate elliptic equations. Math. Comp. 69 (2000) $41-63$.

[8] K.O. Friedrichs, The identity of weak and strong extensions of differential operators. Trans. Amer. Math. Soc. 55 (1944) $132-151$.

[9] J.-L. Lions and E. Magenes, Problèmes aux limites non homogènes et applications. Vol. I and II. Dunod, Paris (1968).

[10] A. Pazy, Semi-groups of linear operators and applications to partial differential equations. Appl. Math. Sci.. 44, Springer Verlag (1983).

[11] O. Pironneau and F. Hecht, FREEFEM. www.ann.jussieu.fr

[12] O. Pironneau and F. Hecht, Mesh adaption for the Black and Scholes equations. East-West J. Numer. Math. 8 (2000) 25-35.

[13] M.H. Protter and H.F. Weinberger, Maximum principles in differential equations. Springer-Verlag, New York (1984). Corrected reprint of the 1967 original.

[14] E. Stein and J. Stein, Stock price distributions with stochastic volatility: an analytic approach. The review of financial studies 4 (1991) 727-752.

[15] H.A Van Der Vorst, Bi-cgstab: a fast and smoothly converging variant of bi-cg for the solution of nonlinear systems. SIAM J. Sci. Statist. Comput. 13 (1992) 631-644.

[16] P. Willmott, J. Dewynne and J. Howison, Option pricing: mathematical models and computations. Oxford financial press (1993). 